\title{
Classification of Paragroup Actions on Subfactors
}

\author{
Dedicated to Professor Masamichi Takesaki on his sixtieth birthday
}

By

\author{
Yasuyuki KAWAHIGASHI
}

\begin{abstract}
Abstræct
We define "a crossed product by a paragroup action on a subfactor" as a certain commuting square of type $\mathrm{II}_{1}$ factors and give their complete classification in a strongly amenable case (in the sense of S. Popa) in terms of a new combinatorial object which generalizes Ocneanu's paragroup.

As applications, we show that the subfactor $N \subset M$ of Goodman-de la Harpe-Jones with index $3+\sqrt{3}$ is not conjugate to its dual $M \subset M_{1}$ by showing the fusion algebras of $N-N$ bimodules and $M$ $M$ bimodules are different, although the principal graph and the dual principal graph are the same. We also make an analogue of the coset construction in RCFT for subfactors in our settings.
\end{abstract}

\section{$\S 1$. Introduction}

Our aim in this paper is to introduce a notion of a "crossed product by a paragroup action on a subfactor", which is a certain commuting square of type $\mathrm{II}_{1}$ factors, and classify them in terms of a combinatorial invariant generalizing Ocneanu's paragroup [35]. Roughly speaking, the standard axioms of paragroups together with a new axiom, the intertwining Yang-Baxter equation, characterize our "canonical commuting cubes" which have information enough to recover the original commuting squares by Popa'a deep theorem [44].

We will discuss relations among our new settings, Rational Conformal Field Theory (RCFT) and 3-dimensional Topological Quantum Field Theory (TQFT). Our new machinery also turns out to be useful for studies of ordinary subfactors, and as applications, we will determine the fusion rules and the TQFT of the subfactor of Goodman-de la Harpe-Jones [16] with index $3+\sqrt{3}$, and initiate the coset construction for subfactors, which is an analogue of the coset construction in RCFT.

Since the pioneering work of V. F. R. Jones [21] on subfactors and his celebrated knot invariant [22], the theory of operator algebras has experienced

Communicated by H. Araki, February 24, 1994.

1991 Mathematics Subject Classification: 46L37

*Department of Mathematical Sciences, University of Tokyo, Komaba, Tokyo 153, Japan. 
unexpected interactions with low dimension topology, quantum group theory, solvable lattice model theory, and conformal field theory. From the operator algebraic viewpoint, these interactions take place on combinatorial level of subfactor theory, and the best theory so far in this point is Ocneanu's paragroup theory [35]. Unfortunately he has not published details of his fundamental theory, but through many efforts [11], [24], [25], [27], [65] and Ocneanu's several lectures [36], [37], [38], the basics of the theory have been now fairly wellunderstood. For analytic aspects of the classification, S. Popa solved the finite depth case first in [43], and later the strongly amenable case in [44] in the ultimate form.

A work of de Boer-Goeree [6] clarified a relation between subfactors and RCFT, and work on the orbifold construction [10], [24], [63], [64] revealed a further deep relation.

A relation between subfactors and 3-dimensional topology was clear from the beginning [22]. E. Witten [62] proposed a general TQFT based on physical idea, and a mathematically rigorous form was given by [49]. Another formulation [55] based on triangulation of 3-manifolds [1] and the quantum $\|_{q}\left(s l_{2}\right) 6 j$-symbols of Kirillov-Reshetikhin [28] also appeared. Ocneanu claimed a general 3-dimensional TQFT of Turaev-Viro type arising from a subfactor of finite depth and a converse construction in [38] and a detailed account was given in [11].

We first give a rather abstract motivation for our work in this paper. On one hand, Ocneanu's basic idea for the paragroup theory [35] was to regard a subfactor $N \subset M$ as a crossed product by an action of a paragroup on $N$. In this way, a paragroup is regarded as a "quantization" of an ordinary group. A recent deep analytic result [47], [48] by S. Popa further strengthens this viewpoint.

On the other hand, there have been studies of group actions on subfactors [7], [23], [26], [29], [31], [45], [46], [59], [60], [61]. We regard "a paragroup action" on a factor as an action of a quantum structure on a classical object, and regard a group action on a subfactor as an action of a classical structure on a quatum object. This suggests that there should be something to be called a paragroup action on a subfactor.

In the theory of ordinary subfactors, what we have is a "crossed products of a paragroup action", so in our setting, we also look for something to be called a "crossed products of a paragroup action on a subfactor". Then we realize that a certain commuting square of type $\mathrm{II}_{1}$ factors

$$
\begin{array}{ccc}
M_{00} & \subset M_{01} \\
\cap & & \cap \\
M_{10} & \subset M_{11}
\end{array}
$$


should be a "crossed products of a paragroup action on a subfactor". We regard $M_{10} \subset M_{11}$ as a crossed product by a paragroup action on a subfactor $M_{00} \subset M_{01}$ and also regard $M_{01} \subset M_{11}$ as a crossed product by a paragroup action on a subfactor $M_{00} \subset M_{10}$.

Next we give more concrete motivations for our work. We initiated the orbifold construction for subfactors in [10], [24] based on an idea in solvable lattice model theory [14], [15], [30]. In this method, we construct an action of a finite group $G$ on a subfactor $N \subset M$ and make a simultaneous fixed point algebras $N^{G} \subset M^{G}$ (or simultaneous crossed product algebras $N \times G \subset M \times G$.) First this method in [24] was used to construct a subfactor of type $D_{2 n}$ from a subfactor of type $A_{4 n-3}$. Later this action on the subfactors of type $A_{4 n-3}$ was identified in [7], [27] with the $\mathbb{Z}_{2}$ actions appearing in descendent sectors in Izumi's work [18] and the general case was clarified by [63], [13]. Study of these actions can be regarded in a sense as a study of the commuting squares.

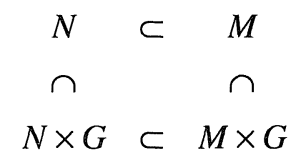

For example, Popa's notion of the co-standard graph [45], [46] can be naturally generalized from this view point. That is, we regard a general commuting square

$$
\begin{array}{ccc}
M_{00} & \subset & M_{01} \\
\cap & & \cap \\
M_{10} & \subset M_{11}
\end{array}
$$

(with appropriate properties) as a "quantization" of the above commuting square (1).

We have another motivation as follows. S. Okamoto recently considered a problem when the commuting square

$$
\begin{array}{cccc}
M_{00} & \subset & M_{01} \\
\cap & & \cap \\
M_{10} & \subset & M_{11}
\end{array}
$$

of approximately finite dimensional (AFD) type $\mathrm{II}_{1}$ factors with $M_{00} \subset M_{11}$ having finite index and finite depth is of the following type:

$$
\begin{array}{ccc}
N \otimes P & \subset & M \otimes P \\
\cap & & \cap \\
N \otimes Q & \subset & M \otimes Q
\end{array}
$$

Here $N \subset M$ and $P \subset Q$ are subfactors of AFD type $\mathrm{II}_{1}$ factors with the finite index and finite depth. Unfortunately, his manuscript contained an error, and he 
could not fix it. From our viewpoint, this problem can be regarded as a "splitting" of a paragroup action on a subfactor. That is, suppose that the subfactor $N \subset M$ in the above commuting square (1) has the relative McDuff property [3] $(N \subset M) \cong(N \otimes R \subset M \otimes R)$, where $R$ is a copy of the AFD type II $_{1}$ factor. If the action $\alpha$ of $G$ splits as id $\otimes \sigma$ on $N \otimes R \subset M \otimes R$, where $\sigma$ is the model action of a finite group $G$ on $R$ [20], then the commuting square becomes

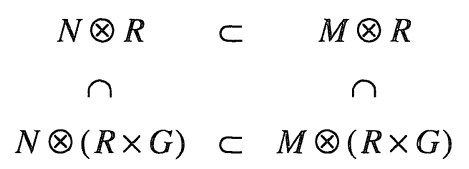

For discrete amenable group actions on single McDuff factors, the right condition for "splitting" is approximate innerness and central freeness as in [8], [34]. In a subfactor setting, approximate innerness was studied in [31], and central freeness was studied in [27], [45], [46]. Thus we expect that the right condition for Okamoto's problem should be "approximate innerness" and "strong outerness" in an appropriate sense. In Section 3, we will give a solution to this problem.

With all the above considerations, we now give the right framework of our theory. We always work under the following assumption in this paper.

Assumption 1.1. The four algebras $M_{00}, M_{01}, M_{10}, M_{11}$ are type $\mathrm{II}_{1}$ factors with the following properties.

1. The square

$$
\begin{array}{ccc}
M_{00} & \subset M_{01} \\
\cap & & \cap \\
M_{10} & \subset M_{11}
\end{array}
$$

is commuting and co-commuting in the sense of [52, Definition 3.4].

2. $\left[M_{11}: M_{00}\right]<\infty$.

3. The subfactor $M_{00} \subset M_{11}$ is extremal and strongly amenable in the sense of [44].

We regard this commuting square as a "crossed product by a paragroup action on a subfactor".

Condition 1 is equivalent to the condition that the above is a commuting square and $\left[M_{11}: M_{10}\right]=\left[M_{01}: M_{00}\right]$ by $[52$, Corollary 7.1$]$. In this case, the commuting square is non-degenerate in the sense of $[44,1.1 .5]$.

In many concrete cases, we further assume that $M_{00} \subset M_{11}$ is of finite depth and all the type $\mathrm{II}_{1}$ factors are approximately finite dimensional (AFD). Note that it was proved in [56] that if $M_{00} \subset M_{01}$ and $M_{01} \subset M_{11}$ have finite depth in the above situation, then $M_{00} \subset M_{11}$ also has finite depth. (For an easier proof, see 
Lemma 3.2 below.) Commuting squares of type $\mathrm{II}_{1}$ factors have been studied in [52], [51], [57], [56] in more abstract settings.

Also note that in general $N \subset P$ and $P \subset M$ are extremal if and only if $N \subset M$ is extremal ([44, 1.2.5(iv)]).

We will give a complete classification of the above type of commuting squares of type $\mathrm{II}_{1}$ factors in terms of combinatorial invariants, and give applications later.

In Section 2, we state the axioms for our new combinatorial system generalizing Ocneanu's paragroup.

In Section 3, we give a bijective correspondence between the above type of commuting squares and our system satisfying the axioms.

In Section 4, we show that RCFT gives non-trivial examples satisfying our axioms.

In Section 5, we apply our theory to study of subfactors of Goodman-de la Harpe-Jones [16]. This shows that our theory is also useful for studies of ordinary subfactors.

In Section 6, we make the coset construction for subfactors as an analogue of the coset construction in RCFT.

In an early stage of the preparation of this work, conversations with $D$. Bisch, D. E. Evans, V. F. R. Jones, S. Okamoto, S. Popa, and F. Xu were inspiring, and around the end of the preparation, comments of U. Haagerup, $M$. Izumi, H. Kosaki, A. Ocneanu, T. Sano, and Y. Watatani were useful. The author thanks for the help of these people.

\section{§2. Combinatorial Axioms for Paragroup Actions on Subfactors and Triple Sequemce of String Algebras}

In this section, we list the combinatorial axiomatization for paragroup actions on subfactors, which generalizes Ocneanu's paragroup in [35]. Our object is a set $\left(\mathscr{G}, \tau, \mu, \imath, \beta_{1}, \beta_{2}, W\right)$ of a connected unoriented graph $\mathscr{G}$, a map $\tau$ from a subset of the vertices of $\mathscr{G}$ onto itself, a real-valued function $\mu$ on the vertices of the graph $\mathscr{G}$, a map $\imath$ defined on a subset of edges of the graph $\mathscr{G}$ onto itself, positive numbers $\beta_{1}, \beta_{2}$ matrices $U_{1}, U_{2}$, and a "connection" $W$ on the graph $\mathscr{G}$. We do not assume that the graph $\mathscr{G}$ is finite, but assume that $\mathscr{G}$ is locally finite in the sense that the number of edges connected to each vertex of $\mathscr{G}$ is finite.

The vertices of the graph $\mathscr{G}$ is a disjoint union of the eight sets ${ }_{1 j} \mathscr{k l}$, where

$$
\begin{aligned}
(i, j, k, l)= & (0,0,0,0),(0,0,0,1),(1,1,0,0),(1,1,0,1), \\
& (0,0,1,0),(0,0,1,1),(1,1,1,0),(1,1,1,1) .
\end{aligned}
$$


We write $i_{i, j}^{\prime, J^{\prime}} \mathscr{G}_{k, l}^{k^{\prime} l^{\prime}}$ for the graph whose vertices is the union $\mathscr{I}_{i, J} \mathscr{G}_{k, l} \cup_{i^{\prime}, J^{\prime}} \mathscr{G}_{k^{\prime}, l^{\prime}}$ and whose edges are the edges of $\mathscr{G}$ connecting a vertex in ${ }_{l, j} \mathscr{G}_{k, l}$ to a vertex in ${ }_{i^{\prime}, l^{\prime}} \mathscr{G}_{k^{\prime}, l^{\prime}}$ where

$$
\begin{aligned}
\left(i^{\prime}, j^{\prime}, k^{\prime}, l^{\prime}, i, j, k, l\right)= & (0,0,0,0,0,0,0,1),(0,0,0,1,1,1,0,1), \\
& (0,0,0,0,1,1,0,0),(1,1,0,0,1,1,0,1), \\
& (0,0,1,0,0,0,1,1),(0,0,1,1,1,1,1,1), \\
& (0,0,1,0,1,1,1,0),(1,1,1,0,1,1,1,1), \\
& (0,0,0,0,0,0,1,0),(0,0,0,1,0,0,1,1), \\
& (1,1,0,0,1,1,1,0),(1,1,0,1,1,1,1,1), \\
& (0,0,0,1,0,0,0,0),(1,1,0,1,0,0,0,1), \\
& (1,1,0,0,0,0,0,0),(1,1,0,1,1,1,0,0), \\
& (0,0,1,1,0,0,1,0),(1,1,1,1,0,0,1,1), \\
& (1,1,1,0,0,0,1,0),(1,1,1,1,1,1,1,0), \\
& (0,0,1,0,0,0,0,0),(0,0,1,1,0,0,0,1), \\
& (1,1,1,0,1,1,0,0),(1,1,1,1,1,1,0,1) .
\end{aligned}
$$

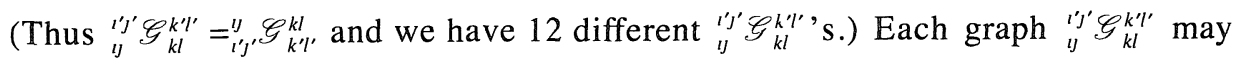
not be connected, but we do require that ${ }_{11}^{00} \mathscr{G}_{00}^{00},{ }_{11}^{00} \mathscr{G}_{01}^{01},{ }_{11}^{00} \mathscr{G}_{10}^{10}$ and ${ }_{11}^{00} \mathscr{G}_{11}^{11}$ be connected. Note that the graph $\mathscr{G}$ looks like a cube whose eight vertices correspond to the eight sets of vertices and whose 12 edges correspond to the 12 sets of edges.

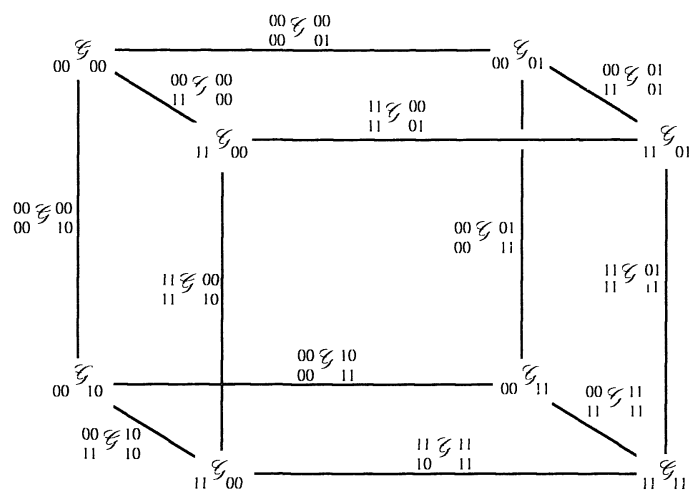

Figure 1.

There are two distinguished vertices $*_{00} \in{ }_{00} \mathscr{G}_{00}, *_{11} \in \in_{11} \mathscr{G}_{11}$. The function $\mu$ assigns a positive numbers $\geq 1$ to each vertex of $\mathscr{G}$ and satisfies $\mu\left(*_{00}\right)=$ $\mu\left(*_{11}\right)=1$.

The first axiom corresponds to the Harmonicity Axiom [35, page 148]. For two vertices $x, y \in \mathscr{G}$, we write $m(x, y)$ for the number of edges of $\mathscr{G}$ connecting $x$ and $y$. 
Axiom 1 (Harmonicity). We have the following identities.

$$
\begin{aligned}
& \beta_{1} \mu(x) \quad=\sum_{v \in y_{i}{ }^{\prime} \bar{k} l} m(x, y) \mu(y), \quad \text { for } x \in{ }_{l j} \mathscr{G}_{k l} \text {, } \\
& \beta_{2} \mu(x)=\sum_{l \in y^{\prime \prime k l}} m(x, y) \mu(y), \quad \text { for } x \in \in_{l,} \mathscr{G}_{k l} \text {, } \\
& \beta_{1} \beta_{2} \mu(x)=\sum_{v \in \in_{i j}^{-j / k l}} m(x, y) \mu(y), \quad \text { for } x \in{ }_{i j} \mathscr{G}_{k l} \text {, }
\end{aligned}
$$

where $\bar{i}=1,0$, for $i=0,1$, respectively.

Note that if the graph $\mathscr{G}$ is finite and each graph ${ }_{l j}^{{ }^{\prime} j^{\prime}} \mathscr{G}_{k l}^{k^{\prime \prime} \prime^{\prime}}$ is connected, the numbers $\beta_{1}, \beta_{2}$ and the function $\mu(\cdot)$ are determined uniquely by the PerronFrobenius theorem.

We require that $\tau$ is a map from ${ }_{00} \mathscr{G}_{00} \cup_{00} \mathscr{G}_{11} \cup_{11} \mathscr{G}_{00} \cup_{11} \mathscr{G}_{11}$ onto itself with order 2. The next axiom corresponds to the Initialization and Parity axioms in [35, page 150].

Axiom 2 (Contragredient map). We require

$$
\begin{aligned}
\tau\left(*_{00}\right) & =*_{00}, \quad \tau\left(*_{11}\right)=*_{11}, \\
\tau\left({ }_{00} \mathscr{G}_{00}\right) & ={ }_{00} \mathscr{G}_{00}, \quad \tau\left({ }_{11} \mathscr{G}_{11}\right)={ }_{11} \mathscr{G}_{11}, \quad \tau\left({ }_{11} \mathscr{G}_{00}\right)={ }_{00} \mathscr{G}_{11},
\end{aligned}
$$

and that

$$
\begin{aligned}
\tau \cdot{ }_{11}^{00} \mathscr{G}_{00}^{00} \cdot \tau & ={ }_{00}^{00} \mathscr{G}_{01}^{00} \cdot{ }_{00}^{00} \mathscr{G}_{11}^{01}={ }_{00}^{00} \cdot \mathscr{G}_{10}^{00} \cdot{ }_{00}^{00} \mathscr{G}_{11}^{10}, \\
\tau \cdot{ }_{00}^{11} \mathscr{G}_{11}^{11} & ={ }_{11}^{11} \mathscr{G}_{01}^{11} \cdot{ }_{11}^{11} \mathscr{G}_{00}^{01}={ }_{11}^{11} \mathscr{G}_{10}^{11} \cdot{ }_{11}^{11} \mathscr{G}_{00}^{10} .
\end{aligned}
$$

In the above formula, the identity $\tau \cdot{ }_{11}^{00} \mathscr{G}_{00}^{00} \cdot \tau={ }_{00}^{00} \mathscr{G}_{01}^{00} \cdot{ }_{00}^{00} \mathscr{G}_{11}^{01}$ means that for $x \in{ }_{00} \mathscr{G}_{00}$ and $y \in \in_{00} \mathscr{G}_{11}$, we get $m(\tau(x), \tau(y))=\sum_{:_{\epsilon_{00}} \text { " } 01} m(x, z) m(z, y)$.

A cell $\left(\xi_{1}, \xi_{2}, \xi_{3}, \xi_{4}\right)$ is a quadruple of edges of the graph $\mathscr{G}$ with $s\left(\xi_{1}\right)=a, \quad r\left(\xi_{1}\right)=b, \quad s\left(\xi_{2}\right)=b, \quad r\left(\xi_{2}\right)=c, \quad s\left(\xi_{3}\right)=d, \quad r\left(\xi_{3}\right)=c, \quad s\left(\xi_{4}\right)=a, \quad r\left(\xi_{4}\right)=d$, where $a, b, c, d$ are four vertices of $\mathscr{G}$ in mutually different ${ }_{\nu} \mathscr{G}_{k l}$ 's. The complexvalued map $W$ is defined on the set of cells, and called a connection. We also use the following symbolic notation for $W\left(\xi_{1}, \xi_{2}, \xi_{3}, \xi_{4}\right)$.

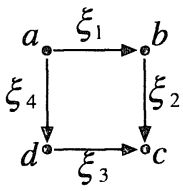


We sometimes drop the labels for edges or vertices if no confusion arises. We also use the convention that if $\left(\xi_{1}, \xi_{2}, \xi_{3}, \xi_{4}\right)$ is not a cell, the above symbol denotes the number 0 .

The bi-unitarity axiom on $W$ in the paragroup setting in [35, page 151] is split into the two parts as in $[24, \S 1]$. The first "half" corresponds to the following axiom in our settings. We write $v(\xi)$ for the set of two vertices of an edge $\xi$ of the graph $\mathscr{G}$.

Axiom 3 (Unitarity)。Choose four edges $\xi_{1}, \xi_{2}, \xi_{1}^{\prime}, \xi_{2}^{\prime}$ of the graph $\mathscr{G}$ with $v\left(\xi_{1}\right)=\{a, b\}, v\left(\xi_{2}\right)=\{b, c\}, v\left(\xi_{1}^{\prime}\right)=\left\{a, b^{\prime}\right\}, v\left(\xi_{2}^{\prime}\right)=\left\{b^{\prime}, c\right\}$, so that $b$ and $b^{\prime}$ belongs to the same ${ }_{1 j} \mathscr{G}_{\mathrm{kl}}$. Suppose that there exist edges $\xi_{3}, \xi_{4}$ of $\mathscr{G}_{\text {such }}$ that $\xi_{1}, \xi_{2}, \xi_{3}, \xi_{4}$ makes a cell. Then we have

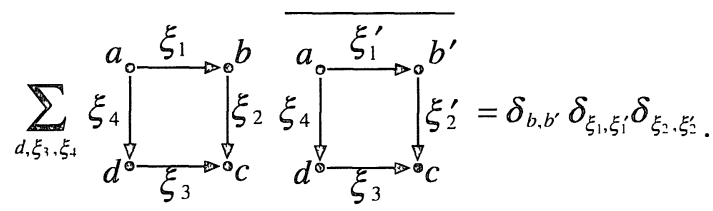

We also have the following axiom which corresponds to the Inversion symmetry axiom and the Rotation symmetry axiom in [35, pages $150-151]$. From our viewpoint, this gives the other "half" of the Bi-unitarity axiom in [35, page 151]. This also corresponds to the crossing symmetry in solvable lattice model theory. Note that our convention of normalizing constant is different form the one in [35] and this is the reason the second formula in the next axiom looks different from the Rotation symmetry [35]. Our convention is compatible with the ones in [10], [11], [19], [24], [25], [37].

Axiom 4 (Renormalization). For a cell $\left(\xi_{1}, \xi_{2}, \xi_{3}, \xi_{4}\right)$, we have the following two identities.
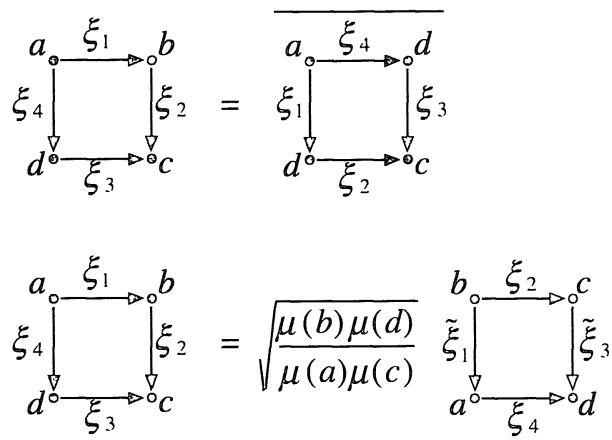

Note that the above imply the following, which looks more familiar. 

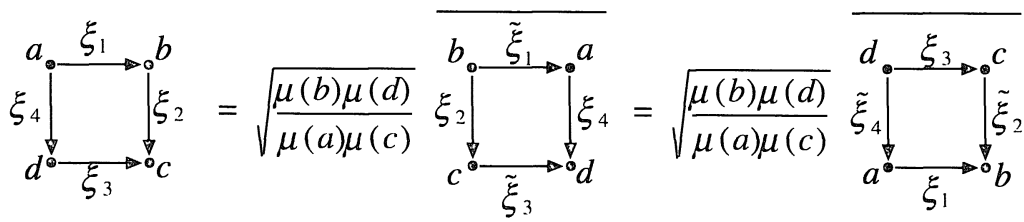

We also use the following (standard) convention for the symbols.

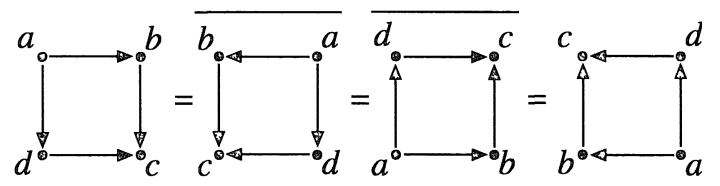

Note that here we dropped the labels for edges for simplicity.

Next we work on an analogue of a partition function as in [35], [37], [24]. We choose one of $*_{00}$ and $*_{11}$ and write $*$ for it. We choose four sequences of edges $\left(\xi_{1}, \xi_{2}, \ldots, \xi_{2 n}\right),\left(\xi_{1}^{\prime}, \xi_{2}^{\prime}, \ldots, \xi_{2 n}^{\prime}\right),\left(\eta_{1}, \eta_{2}, \ldots, \eta_{2 n}\right)$, and $\left(\eta_{1}^{\prime}, \eta_{2}^{\prime} \ldots, \eta_{2 n}^{\prime}\right)$, and next four sequence of vertices $\left(a_{0}, a_{1}, \ldots, a_{2 n}\right),\left(a_{0}^{\prime}, a_{1}^{\prime}, \ldots, a_{2 n}^{\prime}\right),\left(b_{0}, b_{1}, \ldots, b_{2 m}\right)$, and $\left(b_{0}^{\prime}, b_{1}^{\prime}, \ldots, b_{2 m}^{\prime}\right)$ with the following properties.

1. $v\left(\xi_{1}\right)=\left\{a_{0}, a_{1}\right\}, v\left(\xi_{2}\right)=\left\{a_{1}, a_{2}\right\}, \ldots, v\left(\xi_{2 n}\right)=\left\{a_{2 n-1}, a_{2 n}\right\}$.

2. $v\left(\xi_{1}^{\prime}\right)=\left\{a_{0}^{\prime}, a_{1}^{\prime}\right\}, v\left(\xi_{2}^{\prime}\right)=\left\{a_{1}^{\prime}, a_{2}^{\prime}\right\}, \ldots, v\left(\xi_{2 n}^{\prime}\right)=\left\{a_{2 n-1}^{\prime}, a_{2 n}^{\prime}\right\}$.

3. $v\left(\eta_{1}\right)=\left\{b_{0}, b_{1}\right\}, v\left(\eta_{2}\right)=\left\{b_{1}, b_{2}\right\}, \ldots, v\left(\eta_{2 n}\right)=\left\{b_{2 n-1}, b_{2 n}\right\}$.

4. $v\left(\eta_{1}^{\prime}\right)=\left\{b_{0}^{\prime}, b_{1}^{\prime}\right\}, v\left(\eta_{2}^{\prime}\right)=\left\{b_{1}^{\prime}, b_{2}^{\prime}\right\}, \ldots, v\left(\eta_{2 n}^{\prime}\right)=\left\{b_{2 n-1}^{\prime}, b_{2 n}^{\prime}\right\}$.

5. $a_{0}=a_{2 n}=a_{0}^{\prime}=a_{2 n}^{\prime}=b_{0}=b_{2 m}=b_{0}^{\prime}=b_{2 m}^{\prime}=*$.

6. In case of $*=*_{00}$, we require $\xi_{h}, \xi_{h}^{\prime} \in \in_{11}^{00} \mathscr{G}_{00}^{00}$, and in case of $*=*_{11}$, we require $\xi_{h}, \xi_{h}^{\prime} \in{ }_{11}^{00} \mathscr{G}_{11}^{11}$. $(1 \leq h \leq 2 n$.)

7. In case of $*=*_{00}$, we require $b_{h}, b_{h}^{\prime} \in{ }_{00} \mathscr{G}_{k l}$ for some $k, l$, and in case of $*=*_{11}$, we require $b_{h}, b_{h}^{\prime} \in_{11} \mathscr{G}_{k l}$ for some $k, l .(0 \leq h \leq 2 m$.)

8. The edges $\eta_{h}$ and $\eta_{h}^{\prime}$ belong to the same $i_{i, j}^{\prime, J^{\prime}} \mathscr{G}_{k l}^{h^{\prime}, l^{\prime}}$.

Then we have the following definition of the "partition function" as in the paragroup case in [35, page 127], [37, II.2], [24, §1]. First, the dotted lines inside the following diagram means the "filling" of the large rectangle with edges from the graph $\mathscr{G}$ and each choice of the edges is called a configuration. Figure 2 means the product of the $4 \mathrm{~nm}$ connection values of a chosen configuration. 


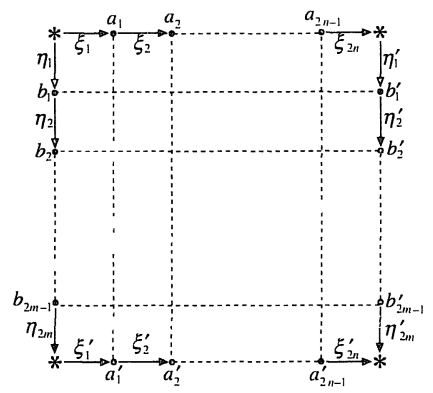

Figure 2.

Then Figure 3 means the value of the sum of the above values over all the possible configurations, which is a direct analogue of a partition function in solvable lattice model theory. (In [35], Ocneanu called this energy.)

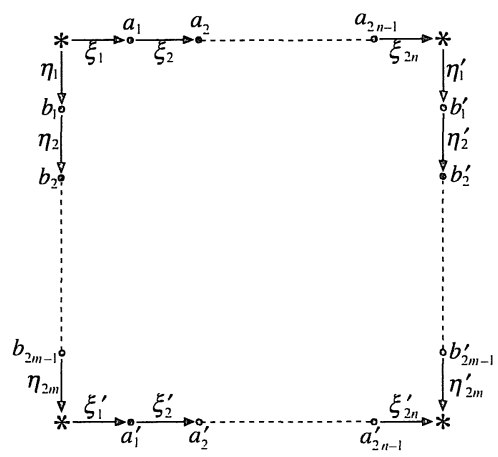

Figure 3. partition function

With this convention, we write the following axiom. This corresponds to the identity $\tau\left(* *_{s}\right)=\tau\left(* *_{*}\right)$ in the Initialization axiom in [35, page 150], but has a more complicated form because later we will not require the trivial relative commutant condition for the subfactor.

Axiom 5 (Initialization). There is an injective map $\imath$ with $\imath^{4}=$ id defined on a subset of edges of the graph $\mathscr{G}$ with the following property. For each $x \in{ }_{11} \mathscr{G}_{00}$, set

$$
\begin{aligned}
& I_{0, x}=\left\{e \in_{11}^{00} \mathscr{G}_{00}^{00} \mid v(e)=\left\{*_{00}, x\right\}\right\}, \\
& I_{1, x}=\left\{e_{1} \cdot e_{2} \mid e_{1} \in_{11}^{11} \mathscr{G}_{11}^{01}, e_{2} \in_{11}^{11} \mathscr{G}_{01}^{00}, *_{11} \in v\left(e_{1}\right), x \in v\left(e_{2}\right), v\left(e_{1}\right) \cap v\left(e_{2}\right) \neq \varnothing\right\}, \\
& I_{2, x}=\left\{e \in_{11}^{00} \mathscr{G}_{11}^{11} \mid v(e)=\left\{*_{11}, \tau(x)\right\}\right\}, \\
& I_{3, x}=\left\{e_{1} \cdot e_{2} \mid e_{1} \in{ }_{00}^{00} \mathscr{G}_{10}^{00} e_{2} \in{ }_{00}^{00} \mathscr{G}_{11}^{10} *_{00} \in v\left(e_{1}\right), \tau(x) \in v\left(e_{2}\right), v\left(e_{1}\right) \cap v\left(e_{2}\right) \neq \varnothing\right\} .
\end{aligned}
$$

Then $l\left(I_{0, x}\right)=I_{1, \lambda} l\left(I_{1, x}\right)=I_{2, x} l\left(I_{2}, x\right)=I_{3, x} l\left(I_{3, x}\right)=I_{0, r}$. Furthermore there is a complex constant $c$ with modulus 1 such that the following identity holds for any $x \in \in_{00} \mathscr{G}_{11}$ and for any $\xi \in \in_{11}^{00} \mathscr{G}_{11}^{11}$ with $s(\xi)=*_{00}$ and $r(\xi)=x$. 


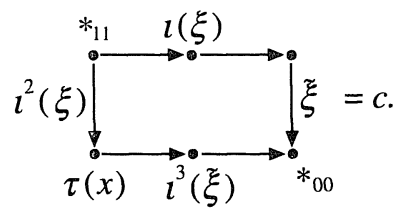

The next axiom is the most important one, the Flatness Axiom, which is an analogue of $[35,(\mathrm{PT})$, page 153$]$.

Axiom 6 (Flatness). We have the identity as in Figure 4.

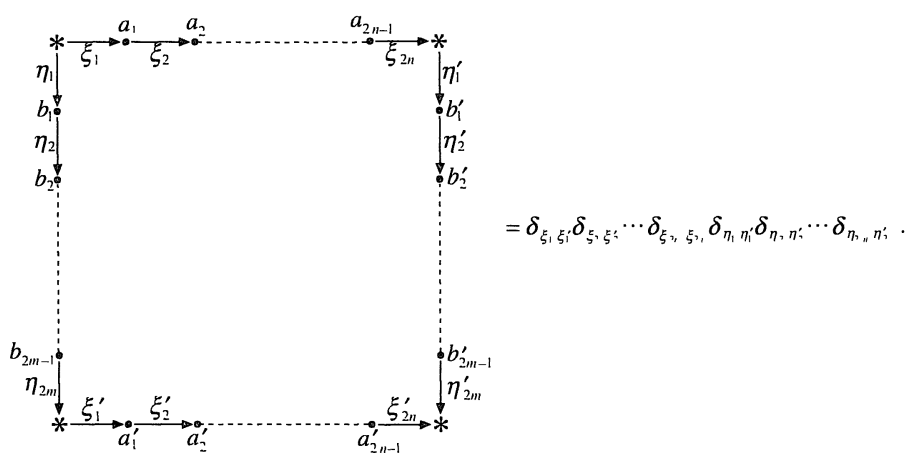

Figure 4. Flatness

Next we need a new axiom which does not correspond to any axiom in the paragroup case. First we choose six edges $\xi_{1}, \xi_{2}, \xi_{3}, \xi_{4}, \xi_{5}, \xi_{6}$ so that they make a hexagon in the graph $\mathscr{G}$ as in Figure 5.

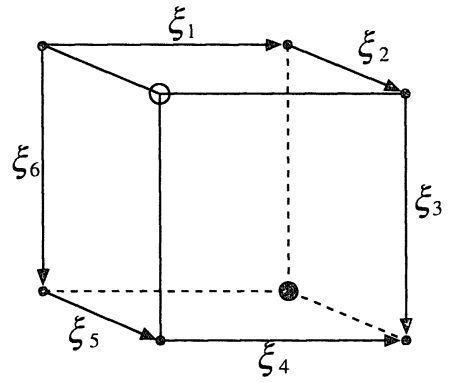

Figure 5.

Then we have the following axiom.

Axiom 7 (Intertwining Yang-Baxter Equation). We have the following identity. 


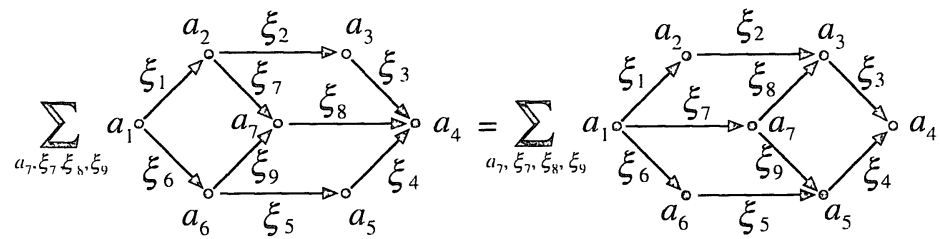

where the both hand sides mean the sum of the products of three connection values over all the possible choices of $a_{7}, \xi_{7}, \xi_{8}, \xi_{9}$ for any fixed choice of $\xi_{1}, \xi_{2}, \xi_{3}, \xi_{4}, \xi_{5}, \xi_{6}$ as above. On the lift hand side, the vertex $a_{7}$ is chosen from the corner marked with $\circ$ in Figure 5, and on the right hand side, it is chosen from the corner 0 .

In the above identity, we used the following convention as in Figure 6.
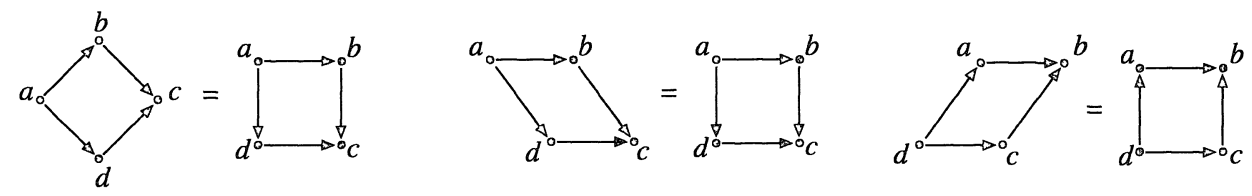

Figure 6.

(We again dropped the labels for edges.)

If the graph $\mathscr{G}$ is finite, which corresponds to the finite depth condition of subfactors, the above system of axioms are enough for our purpose, but we would like to work on subfactors of infinite depth with strong amenability later, so we need an extra axiom for this strong amenability in the sense of [44].

First we construct a triple sequence of string algebras $\left\{A_{j k l}\right\}_{J . k, l \geq 0}$ starting from $*_{00}$ as follows. Choose the starting point $*$ to be $*_{00} \in_{00} \mathscr{G}_{00}$. Construct the three sequences of string algebras $\left\{A_{j 00}\right\}_{J \geq 0},\left\{A_{0 k 0}\right\}_{k \geq 0},\left\{A_{001}\right\}_{l \geq 0}$ starting from $*$ with the graphs ${ }_{11}^{00} \mathscr{G}_{00}^{00},{ }_{00}^{00} \mathscr{G}_{10}^{00},{ }_{00}^{00} \mathscr{G}_{01}^{00}$ respectively. Then use the entire graph $\mathscr{G}$ and the connection $W$ to get the triple sequence as in $[37$, II.2], $[24, \S 1]$. That is, we use the following rule of changes of bases as in [37, II.2], [24, §1].

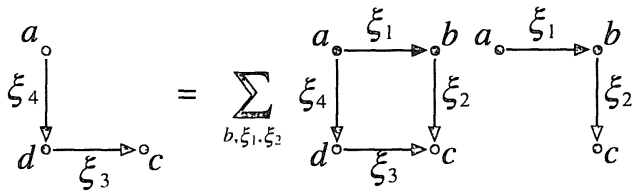

Note that the first part of the sequence looks as in Figure 7. 


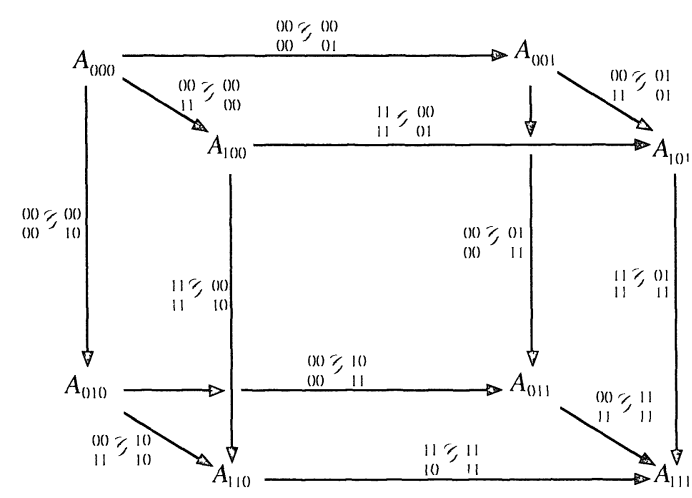

Figure 7.

Here arrows mean embeddings, and we look the graph from the same direction as in Figure 1.

Furthermore, we fix the following identifications of edges. First for any edge $\xi \in \in_{11}^{00} \mathscr{G}_{00}^{00}$ connecting $*_{00}$ and $x \in \in_{11} \mathscr{G}_{00}$, we identify it with a pair of edges $\xi^{\prime} \in{ }_{11}^{11} \mathscr{G}_{11}^{01}$ and $\xi^{\prime \prime} \in{ }_{11}^{11} \mathscr{G}_{01}^{00}$ with $\imath(\xi)=\xi^{\prime} \cdot \xi^{\prime \prime}$. Second for any edge $\xi \in{ }_{11}^{00} \mathscr{G}_{11}^{11}$ connecting $*_{11}$ and $x \in \in_{00} \mathscr{G}_{11}$, we identify it with a pair of edges $\xi^{\prime} \in \in_{00}^{00} \mathscr{G}_{10}^{00}$ and $\xi^{\prime \prime} \in_{00}^{00} \mathscr{G}_{11}^{10}$ with $\imath(\xi)=\xi^{\prime} \cdot \xi^{\prime \prime}$. Then as in [25, pages 134-135], we can extend the definition of the string algebras $A_{j k l}$ to the case $j, k, l \in \mathbb{Z}, k \geq-j, l \geq-j$.

We define a normalized trace $\operatorname{tr}$ on $\cup_{j h l} A_{\text {jhl }}$ as follows. For a string $\left(\xi_{+}, \xi_{-}\right) \in A_{j l}$, we define $\operatorname{tr}\left(\left(\xi_{+}, \xi_{-}\right)\right)=\beta_{1}^{-J-h} \beta_{2}^{-j-l} \mu\left(r\left(\xi_{+}\right)\right)$, the notation $r\left(\xi_{+}\right)$denotes the endpoint of the path $\xi_{+}$(and $s\left(\xi_{+}\right.$) denotes the starting point of $\xi_{+}$). By Axiom 1 and the embedding rule of the string algebra, this tr is well defined on $\cup_{\jmath k l} A_{\jmath k l}$. Then we define $A_{\infty<l}$ to be the von Neumann algebra obtained by the GNScompletion with respect to tr. We define the vertical Jones projections $e_{h} \in A_{0, k+1, l}$ and $f_{l} \in A_{0, k, l+1}$ as in [37, II.2], [24, §1]. That is, $e_{h}$ is given by the following.

$$
e_{h}=\sum_{|\alpha|=n-1,|v|=|n|=1} \frac{\mu(r(v))^{1 / 2} \mu(r(w))^{1 / 2}}{\beta_{1} \mu(r(\alpha))}(\alpha \cdot v \cdot \tilde{v}, \alpha \cdot w \cdot \tilde{w}),
$$

$\alpha$ is any path from $*$ in the graph ${ }_{00}^{00} \mathscr{G}_{10}^{00}$, and $v, w$ are chosen so that the compositions are possible in ${ }_{00}^{00} \mathscr{G}_{10}^{00}$, and $|\cdot|$ denote the length of a path. We also define the Jones projections $p_{j}$ in $A_{j+1,0,0}$ similarly.

The following corresponds to [44, 1.4 .2 (vii)] and is equivalent to factoriality of the von Neumann algebras $A_{\infty, k l}$.

Axiom 8 (Ergodicity). For the vertices in $00 \mathscr{G}_{1 \mathrm{l}}$, the vector $\mu(\cdot)$ is the unique $\mu$-bounded eigenvector of ${ }_{11}^{00} \mathscr{G}_{1 J}^{\prime \prime}\left({ }_{11}^{00} \mathscr{G}_{1 J}^{\prime \prime}\right)^{t}$ corresponding to the eigenvalue $\beta_{1} \beta_{2}$, where $i, j=0,1$. 
Note that if the graph $\mathscr{G}$ is finite, then this factoriality automatically holds as usual, because the four graphs ${ }_{11}^{00} \mathscr{G}_{01}^{00},{ }_{11}^{00} \mathscr{G}_{11}^{11}, 00 \mathscr{G}_{01}^{01}$, and ${ }_{11}^{00} \mathscr{G}_{10}^{10}$ are connected.

The following corresponds to [44, Theorem 5.3.1(vii)]. (Also see [37, page 35 ], where Ocneanu defines his amenability in a slightly different way from [44].)

Axiom 9 (Amenability). We have the following.

$$
E_{A_{\infty,-1,-1}^{\prime} \cap A_{\infty, \infty, \infty}}\left(p_{1}\right)=E_{A_{\infty,-2,-2}^{\prime} \cap A_{\infty, \infty, \infty}}\left(p_{2}\right)=\beta_{1}^{-2} \beta_{2}^{-2} .
$$

Note that $p_{J}$ is the Jones projection for $A_{J-1, \infty, \infty} \subset A_{J, \infty, \infty}$. This condition means extremality of the model inclusion. See arguments preceding the main theorem in Section 3.

The operator $E_{A_{\infty,-1,-1}^{\prime} \cap A_{\infty, \infty, \infty}}\left(p_{1}\right)$ can be expressed as $\lim _{J} E_{A_{j,-1,-1}^{\prime} \cap A_{\infty, \infty, \infty}}\left(p_{1}\right)$ and there is an explicit formula for $E_{A_{j,-1,-1}^{\prime} \cap A_{\infty, \infty, \infty}}$ in [36], so in principle, the above amenability axiom can be checked by computation.

We now define an equivalence relation between two systems satisfying the above axioms. This is an analogue of the definition in [35, page 154]. (Our convention is slightly different from the one in [35].)

Let $\left(\mathscr{G}, \tau, \mu, \imath, \beta_{1}, \beta_{2}, W\right)$ be a set satisfying the above axioms. A perturbation $u$ of the connection $W$ is a set of unitary matrices $(u(\xi, \eta))_{\xi, \eta}$ associated to each pair of adjacent vertices $x, y$ of $\mathscr{G}$, where $\xi, \eta$ are edges of $\mathscr{G}$ connecting $x$ and $y$. We require that $u(\tilde{\xi}, \tilde{\eta})=\overline{u(\xi, \eta)}$. The perturbed connection $W^{\#}$ is defined by the following formula.

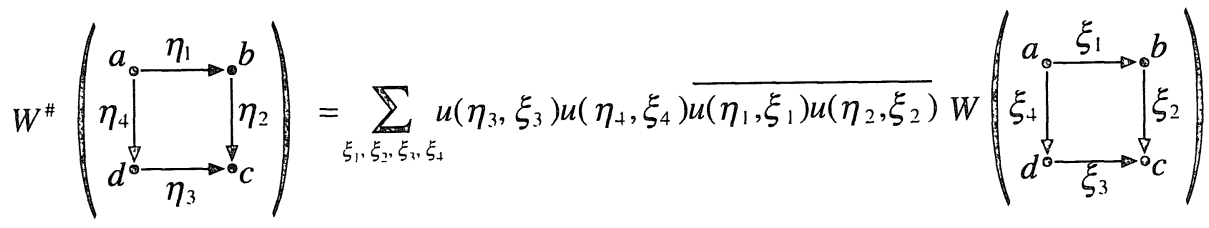

We assume that the Initialization Axiom is preserved. The systems $\left(\mathscr{G}, \tau, \mu, \imath, \beta_{1}, \beta_{2}, W\right)$ and $\left(\mathscr{G}, \tau, \mu, \imath, \beta_{1}, \beta_{2}, W^{\#}\right)$ are called equivalent. We simply write $\left(\mathscr{G}, \tau, \mu, l, \beta_{1}, \beta_{2}, W\right)$ for denoting the equivalence class. Two systems $\left(\mathscr{G}, \tau, \mu, \imath, \beta_{1}, \beta_{2}, W\right)$ and $\left(\mathscr{G}^{\prime}, \tau^{\prime}, \mu^{\prime}, \iota^{\prime}, \beta_{1}, \beta_{2}, W^{\prime}\right)$ are called isomorphic if there is a perturbation $W^{\#}$ of $W$ and a graph isomorphism

$$
\theta: \mathscr{G} \rightarrow \mathscr{G}^{\prime} \text { with } \mu^{\prime} \cdot \theta=\mu, \theta^{-1} \cdot \imath^{\prime} \cdot \theta=\imath, \theta^{-1} \cdot \tau^{\prime} \cdot \theta=\tau \text {, and } W^{\prime} \cdot \theta=W^{\#} \text {. }
$$




\section{§3. From Commuting Squares of Type $\mathbb{I I}_{1}$ Factors to Combinatorial Data and Back}

In this section, we construct combinatorial date satisfying the axioms in $\S 1$ from commuting squares of type $\mathrm{II}_{1}$ factors with Assumption 1.1 and give the converse construction based on bimodule approach. For basics of the bimodule theory over factors, see [11], [38], [42], [65].

For simplicity of notations, we write ${ }_{i j} X_{k l}$ for a bimodule ${ }_{M_{i j}} X_{M_{k l}}$ where $i, j, k, l=0,1$. We also write $\otimes_{l \jmath}$ for the relative tensor $\otimes_{M_{i}}$ for bimodules and $E_{l \jmath}$ for $E_{M_{l j}}$. We often regard von Neumann algebras as bimodules and in such cases we have to take $L^{2}$-completions with respect to the trace, but we often drop $L^{2}()$ for simplicity of notations. For example, ${ }_{00}\left(M_{00}\right)_{00}$ means the $M_{00}-M_{00}$ bimodule $L^{2}\left(M_{00}\right)$.

Lemma 3.1. Under Assumption 1.1, we have natural isomorphisms $M_{01} \otimes_{00} M_{10} \cong M_{11}$ as $M_{01}-M_{10}$ bimodules, and $M_{10} \otimes_{00} M_{01} \cong M_{11}$ as $M_{10}-M_{01}$ bimodules.

Proof. We define a map $\pi: M_{01} \otimes_{00} M_{10} \rightarrow M_{11}$ by $\pi\left(x \otimes_{00} y\right)=x y$ for $x \in M_{01}, y \in M_{10}$. For $x_{1}, x_{2} \in M_{01}$ and $y_{1}, y_{2} \in M_{10}$, we get

$$
\begin{aligned}
\left(x_{1} \otimes_{00} y_{1}, x_{2} \otimes_{00} y_{2}\right) & =\left(x_{1} E_{00}\left(y_{1} y_{2}^{+}\right), x_{2}\right) \\
= & \operatorname{tr}\left(x_{2}^{\circ} x_{1} E_{00}\left(y_{1} y_{2}^{\prime}\right)\right) \\
= & \operatorname{tr}\left(x_{2}^{\prime} x_{1} y_{1} y_{2}^{\circ}\right) \\
= & \left(\pi\left(x_{1} \otimes_{00} y_{1}\right), \pi\left(x_{2} \otimes_{00} y_{2}\right)\right),
\end{aligned}
$$

where we used the commuting square condition $E_{00}=E_{10}$ on $M_{01}$. Thus $\pi$ extends to an isometry and it is surjective by [52, Corollary 7.1]. The other isomorphism is proved similarly.

Q.E.D.

Next we make basic constructions vertically and horizontally from the initial commuting square, and get a double sequence of type $\mathrm{II}_{1}$ factors $M_{k l}$ as in [52, Section 7]. Note that the square

$$
\begin{array}{ccc}
M_{k l} & \subset & M_{k, l+1} \\
\cap & & \cap \\
M_{k+1, l} & \subset & M_{k+1, l+1}
\end{array}
$$

is again commuting and co-commuting. Then the increasing sequence

$$
M_{00} \subset M_{11} \subset M_{22} \subset M_{33} \subset \cdots
$$


is the Jones tower of the subfactor $M_{00} \subset M_{11}$, and for this subfactor, we choose a tunnel

$$
\cdots \subset M_{-2,-2} \subset M_{-1,-1} \subset M_{00} \subset M_{11} .
$$

Here we give a simple proof of a theorem in [56], which was mentioned in the Introduction.

Lemma 3.2. Under Conditions 1, 2 of Assumption 1.1, the following are equivalent.

1. $M_{00} \subset M_{11}$ has finite depth.

2. $M_{00} \subset M_{01}$ and $M_{00} \subset M_{10}$ have finite depth.

Proof. We have ${ }_{00}\left(M_{n n}\right)_{00}={ }_{00}\left(M_{n 0} \otimes_{00} M_{0 n}\right)_{00}$ by the above lemma. So ${ }_{00}\left(M_{n n}\right)_{00}$ has only finitely many mutually non-isomorphic irreducible components if and only if both of ${ }_{00}\left(M_{n 0}\right)_{00}$ and ${ }_{00}\left(M_{0 n}\right)_{00}$ have only finitely many mutually non-isomorphic irreducible components.

Q.E.D.

Note that the above form looks slightly different from the theorem in [56], but both are equivalent. For example, if $M_{00} \subset M_{01}$ and $M_{01} \subset M_{11}$ have finite depth, then $M_{01} \subset M_{02}$ has finite depth, so by the above lemma, $M_{01} \subset M_{12}$ has finite depth, then $M_{-1,0} \subset M_{12}$ has finite depth, which implies that $M_{00} \subset M_{11}$ has finite depth.

Next we construct a triple sequence $\left(X_{j l}\right)$ of bimodules inductively as follows. Let $X_{000}={ }_{00}\left(M_{00}\right)_{00}$. If $k, l$ are even, then set $X_{, \cdot h \cdot l+1}=X_{j h l} \otimes_{00}\left(M_{01}\right)_{01}$ and $X_{J, k+1, l}=X_{j k l} \otimes_{01}\left(M_{11}\right)_{11}$. If $k$ is even and $l$ is odd, then set $X_{J, k, l+1}=X_{j k l} \otimes_{01}\left(M_{01}\right)_{00}$ and $X_{J, k+1, l}=X_{j k l} \otimes_{01}\left(M_{11}\right)_{11}$. If $k$ is odd and $l$ is even, then set $X_{J, k, l+1}=X_{j k l} \otimes_{10}\left(M_{11}\right)_{11}$ and $X_{J, k+1, l}=X_{j k l} \otimes_{10}\left(M_{10}\right)_{00}$. If $k, l$ are odd, then set $X_{J, k, l+1}=X_{j k l} \otimes_{11}\left(M_{11}\right)_{10}$ and $X_{J, k+1, l}=X_{j k l} \otimes_{11}\left(M_{11}\right)_{01}$. If $j$ is even, then set $X_{j+1, k, l}={ }_{11}\left(M_{11}\right) \otimes_{00} X_{j k l}$, and if $j$ is odd, then set $X_{j+1, k, l}={ }_{00}\left(M_{11}\right) \otimes_{11} X_{j l l}$. With natural isomorphisms

$$
\begin{aligned}
& { }_{00}\left(M_{01} \otimes_{01} M_{11}\right)_{11} \cong_{00}\left(M_{11}\right)_{11} \cong_{00}\left(M_{10} \otimes_{10} M_{11}\right)_{11}, \\
& { }_{01}\left(M_{01} \otimes_{00} M_{10}\right)_{10} \cong_{01}\left(M_{11}\right)_{10} \cong_{01}\left(M_{11} \otimes_{11} M_{11}\right)_{10}, \\
& { }_{10}\left(M_{10} \otimes_{00} M_{01}\right)_{01} \cong_{10}\left(M_{11}\right)_{01} \cong_{10}\left(M_{11} \bigotimes_{11} M_{11}\right)_{01}, \\
& { }_{11}\left(M_{11} \otimes_{10} M_{10}\right)_{00} \cong_{11}\left(M_{11}\right)_{00} \cong_{11}\left(M_{11} \otimes_{01} M_{01}\right)_{00},
\end{aligned}
$$

we know that this construction is compatible. Thus $X_{j k l}$ is an $M_{[j][J]} M_{[k][l]}$ bimodule, where [j] denotes 0 [resp. 1] when $j$ is even [resp. odd].

We set $B_{j k l}=\operatorname{End}\left(X_{j l}\right)$, where $\operatorname{End}\left(X_{j l}\right)$ means the set of bounded linear maps on the Hilbert space $X_{j k l}$ which commute with the left and right actions of 
the type $\mathrm{II}_{1}$ factors. Note that this is isomorphic to $M_{-J,-J}^{\prime} \cap M_{k l}$ by [41] and thus finite dimensional. If $k, l$ are even, we regard $B_{J l l}$ as a subalgebra of $B_{J, k, l+1}$ with the embedding $\xi \in \operatorname{End}\left(X_{\jmath k l}\right) \mapsto \xi \otimes_{00} \mathrm{id}_{00\left(M_{01}\right)_{01}} \in \operatorname{End} X_{J . k . l+1}$ and define the other embeddings similarly. Because the embeddings are compatible, we get a triple increasing sequence of finite dimensional algebras $B_{j k l}$. By looking at the isomorphism between each $B_{j k l}$ and each $M_{-J,-J}^{\prime} \cap M_{k l}$ and the embeddings of the both triple sequences, we can conclude that the sequence $\left(B_{j k l}\right)$ and the sequence of the higher relative commutants $\left(M_{-J,-j}^{\prime} \cap M_{k l}\right)$ are isomorphic, as in the ordinary paragroup case. (For the ordinary paragroup case, see an exposition [27], for example.)

We make irreducible decompositions of the bimodules $X_{2, .2 h, 2 l}$ to get a set of (isomorphism classes) of all the $M_{00}-M_{00}$ bimodules arising in this way. Set this to be the vertex set ${ }_{00} \mathscr{\%}_{00}$. Similarly, we make vertex sets ${ }_{00} \mathscr{\%}_{01},{ }_{00} \xi_{10},{ }_{00} \xi_{11}$, ${ }_{11} \mathscr{G}_{00},{ }_{11} \mathscr{G}_{01},{ }_{11} \mathscr{G}_{10}$, and ${ }_{11} \mathscr{G}_{11}$ from the irreducible decompositions of the bimodules $X_{2 \jmath, 2 h, 2 l+1}, X_{2, .2 h+1,2 l}, \quad X_{2 \jmath, 2 h+1,2 l+1}, X_{2 \jmath+1,2 h, 2 l}, X_{2 \jmath+1,2 h, 2 l+1}, X_{2 \jmath+1,2 h+1,2 l}$ and $X_{2 \jmath+1,2 h+1,2 l+1}$ respectively. For an $M_{11}-M_{00}$ bimodule $X \in_{00}, \mathscr{G}_{00}$ and an $M_{00}-M_{00}$ bimodule $Y \in \in_{11} \mathscr{G}_{00}$, we make edges in ${ }_{11}^{00} \mathscr{G}_{00}^{00}$ so that their number is equal to the multiplicity of $Y$ in ${ }_{11}\left(M_{11} \otimes_{00} X\right)_{00}$. Note that by Frobenius reciprocity in [38], [65], the number of edges is also equal to the multiplicity of $X$ in ${ }_{00}\left(M_{11} \otimes_{11} Y\right)_{00}$. We similarly make edges for the other parts of $\mathscr{G}$. Note that ${ }_{11}^{00} \mathscr{G}_{00}^{00}$ and ${ }_{11}^{00} \mathscr{G}_{11}^{11}$ are connected because these are the principal graph and the dual principal graph of the subfactor $M_{00} \subset M_{11}$. If this subfactor $M_{00} \subset M_{11}$ is of finite depth, our graph $\mathscr{G}$ is finite. In general, $\mathscr{G}$ may be infinite, but it is always locally finite because the index $\left[M_{11}: M_{00}\right]$ is finite. We set $*_{00}={ }_{00}\left({ }_{00} M_{00}\right)_{00}$ and $*_{11}={ }_{11}\left({ }_{11} M_{11}\right)_{11}$ for a bimodule ${ }_{1} X_{k l}$ in the vertex set of $\mathscr{G}$, we define the function $\mu$ by $\mu\left({ }_{l j} X_{k l}\right)=\sqrt{\operatorname{dim}_{l j} X \operatorname{dim} X_{k l}}$, where $i, j, k, l=0,1$. Because we assume extremality of the subfactor $M_{00} \subset M_{11}$, we have $\mu\left({ }_{00} X_{00}\right)=\operatorname{dim}{ }_{00} X=\operatorname{dim} X_{00}, \mu\left({ }_{00} X_{01}\right)$ $=\left[M_{01}: M_{00}\right]^{-1 / 2} \operatorname{dim}{ }_{00} X$, and other similar equalities. From these, we get the Harmonicity Axiom.

For $M_{00}-M_{00}$ bimodules, $M_{00}-M_{11}$ bimodules, $M_{11}-M_{00}$ bimodules, $M_{11}-M_{11}$ bimodules, we define $\tau(X)=\bar{X}$, the conjugate bimodule. Then it is clear that we have the axiom on the contragredient map.

We have to define the connection next. For an $M_{00}-M_{00}$ bimodule $X \in_{00} \mathscr{G}_{00}$ and an $M_{11}-M_{00}$ bimodule $Y \in \in_{11} \mathscr{G}_{00}$, suppose that the number of edges connecting $X$ and $Y$ is $n$. Then we choose intertwiners $\xi_{1}, \ldots, \xi_{n} \in \operatorname{Hom}\left({ }_{11}\left(M_{11} \otimes_{00} X\right)_{00},{ }_{11} Y_{00}\right)$ 
so that each is co-isometry and the initial projection of $\xi$, are mutually orthogonal. We have $n$ edges and give them orientations going from $X$ to $Y$. We assign each intertwiner $\xi$, to each edge, and to the same edge with the reverse orientation, we assign its Frobenius dual ${ }^{M_{11}} \xi_{\jmath} \in \operatorname{Hom}\left({ }_{00}\left(M_{11} \otimes_{11} Y\right)_{00},{ }_{00} X_{00}\right)$. (See [38], [65] for the Frobenius dual.) We know that ${ }^{M_{11}} \xi_{J}$ 's are co-isometries and their initial projections are mutually orthogonal. We make similar assignment of co-isometry intertwiners to each edge of the graph $\mathscr{G}$. Take the following square made from $\mathscr{G}$

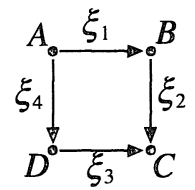

If $A \in_{00} \mathscr{G}_{00}, B \in_{00} \mathscr{G}_{01}, C \in_{11} \mathscr{G}_{01}$, and $D \in_{11} \mathscr{G}_{00}$, then we set $W\left(\xi_{1}, \xi_{2}, \xi_{3}, \xi_{4}\right)=$ $\xi_{3}\left(\xi_{4} \otimes \mathrm{id}_{M_{01}}\right)\left(\mathrm{id}_{M_{11}} \otimes \xi_{1}^{*}\right) \xi_{2}$. If $A \in_{00} \mathscr{G}_{00}, B \in_{00} \mathscr{G}_{01}, C \in_{00} \mathscr{G}_{11}$, and $D \in_{00} \mathscr{G}_{10}$, then we set $W\left(\xi_{1}, \xi_{2}, \xi_{3}, \xi_{4}\right)=\xi_{3}\left(\xi_{4} \otimes \mathrm{id}_{M_{11}}\right)\left(\mathrm{id}_{A} \otimes \pi\right)\left(\xi_{11} \otimes \mathrm{id}_{M_{11}}\right) \xi_{2}^{\prime}$, where $\pi$ is a natural isomorphism from ${ }_{00}\left(M_{01} \otimes_{01} M_{11}\right)_{11}$ to ${ }_{00}\left(M_{10} \otimes_{10} M_{11}\right)_{11}$. In the general case, the square is one of the above two types, so we make a similar definition of $W$ in each case. Then the Unitarity Axiom holds by co-isometry condition of $\xi_{\text {, }}$.

The next axiom is the Renormalization axiom. If the square involving $\xi_{1}, \xi_{2}, \xi_{3}, \xi_{4}$ is of the above first type, then this axiom is just standard Frobenius reciprocity as in [38], [65]. If it is one of the second type, the equality is proved as follows. For simplicity, we assume $A \in_{00} \mathscr{G}_{00}, B \in_{00} \mathscr{G}_{01}, C \in_{00} \mathscr{G}_{11}$, and $D \in_{00} \mathscr{G}_{10}$ as above. In this case, the following square

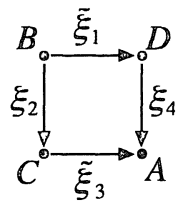

has a value $\xi_{3}^{M_{11}}\left(\xi_{2} \otimes \mathrm{id}_{M_{11}}\right)\left(\mathrm{id}_{B} \otimes \pi^{\prime}\right)\left(\xi_{1}^{M_{01}} \otimes \mathrm{id}_{M_{10}}\right) \xi_{4}$, where $\pi^{\prime}$ is a natural isomorphism from ${ }_{01}\left(M_{01} \otimes_{00} M_{10}\right)_{10}$ to ${ }_{01}\left(M_{11} \otimes_{11} M_{11}\right)_{10}$. We denote this value by $W^{\prime}$ and the value of the original square by $W$. We prove the identity $W^{\prime}=\sqrt{\frac{\mu(A) \mu(C)}{\mu(B) \mu(D)}} \bar{W}$ as follows. We choose a right basis $\left\{d_{l}\right\}_{l}$ for $D_{10}$, a right basis $\left\{x_{\jmath}\right\}$, for $C_{11}$, a right basis $\{1\}$ for $\left(M_{01}\right)_{01}$, and a right basis $\{1\}$ for $\left(M_{11}\right)_{11}$. Using the same notations as in [38], we make the following computations. 
$W^{\prime} \operatorname{dim} D_{10}$

$$
\begin{aligned}
& =\sum_{l}\left(\xi_{3}^{M_{11}}\left(\xi_{2} \otimes \mathrm{id}_{M_{11}}\left(\mathrm{id}_{B} \otimes \pi^{\prime}\right)\left(\xi_{1}^{M_{01}^{4}} \otimes \mathrm{id}_{M_{10}}\right) \xi_{4}^{*} d_{l}, d_{l}\right)\right. \\
& =\sqrt{\frac{\operatorname{dim} A_{00} \operatorname{dim} D_{10}}{\operatorname{dim} B_{01} \operatorname{dim} C_{11}}} \sum_{t}\left(\left(\mathrm{id}_{B} \otimes \pi^{\prime}\right)\left(\tilde{\xi}_{1} \otimes \mathrm{id}_{M_{10}}\right) \xi_{4}^{\dagger} d_{l},\left(\xi_{2}^{\dagger} \otimes \mathrm{id}_{M_{11}}\right) \tilde{\xi}_{3} d_{t}\right) \\
& =\lim _{n} \sqrt{\frac{\operatorname{dim} A_{00} \operatorname{dim} D_{10}}{\operatorname{dim} B_{01} \operatorname{dim} C_{11}}} \sum_{l, k}\left(\left(\mathrm{id}_{B} \otimes \pi^{\prime}\right)\left(\tilde{\xi}_{1} \otimes \mathrm{id}_{M_{10}}\right) a_{t, k}^{(n)} \otimes z_{l, k}^{(n)},\left(\xi_{2}^{+} \xi_{3}\left(d_{t} \otimes 1\right) \otimes 1\right)\right. \\
& =\lim _{n} \sqrt{\frac{\operatorname{dim} A_{00} \operatorname{dim} D_{10}}{\operatorname{dim} B_{01} \operatorname{dim} C_{11}}} \sum_{l, k}\left(\left(\operatorname{id}_{B} \otimes \pi^{\prime}\right)\left(\xi_{1}\left(a_{l, k}^{(n)} \otimes 1\right) \otimes 1 \otimes z_{t, k}^{(n)}\right),\left(\xi_{2}^{r} \xi_{3}\left(d_{l} \otimes 1\right) \otimes 1\right)\right. \\
& =\lim _{n} \sqrt{\frac{\operatorname{dim} A_{00} \operatorname{dim} D_{10}}{\operatorname{dim} B_{01} \operatorname{dim} C_{11}}} \sum_{l, k}\left(\xi_{2}\left(\xi_{1}\left(a_{l, k}^{(n)} \otimes 1\right) \otimes z_{l, k}^{(n)}\right),\left(\xi_{3}\left(d_{l} \otimes 1\right)\right)\right. \\
& =\lim _{n} \sqrt{\frac{\operatorname{dim} A_{00} \operatorname{dim} D_{10}}{\operatorname{dim} B_{01} \operatorname{dim} C_{11}}} \sum_{t, h, J}\left(\xi_{2}\left(\xi_{1}\left(a_{t, k}^{(n)} \otimes 1\right) \otimes z_{t, k}^{(n)}\right), c_{J}\left(c_{J}, \xi_{3}\left(d_{\imath} \otimes 1\right)\right)^{\circ}\right) \\
& =\lim _{n} \sqrt{\frac{\operatorname{dim} A_{00} \operatorname{dim} D_{10}}{\operatorname{dim} B_{01} \operatorname{dim} C_{11}}} \sum_{\imath, h, J}\left(\xi_{2}\left(\xi_{1}\left(a_{t, k}^{(n)} \otimes 1\right) \otimes z_{t, k}^{(n)}\right)\left(d_{\imath} \otimes 1, \xi_{3}^{\prime}\left(c_{J}\right)\right)^{\circ}, c_{\jmath}\right) \\
& =\sqrt{\frac{\operatorname{dim} A_{00} \operatorname{dim} D_{10}}{\operatorname{dim} B_{01} \operatorname{dim} C_{11}}} \sum_{\imath, \mathrm{J}}\left(\xi_{2}\left(\xi_{1} \otimes \mathrm{id}_{M_{10}}\right)\left(\mathrm{id}_{A} \otimes \pi^{\prime}\right)\left(\xi_{4}^{\prime} \otimes \mathrm{id}_{M_{11}}\right)\left(d_{\imath} \otimes 1\right)\left(d_{\imath} \otimes 1, \xi_{3}^{*}\left(c_{\jmath}\right)\right)^{\circ}, c_{\jmath}\right) \\
& =\sqrt{\frac{\operatorname{dim} A_{00} \operatorname{dim} D_{10}}{\operatorname{dim} B_{01} \operatorname{dim} C_{11}}} \sum_{J}\left(\xi_{2}\left(\xi_{1} \otimes \mathrm{id}_{M_{11}}\right)\left(\mathrm{id}_{A} \otimes \pi^{\prime}\right)\left(\xi_{4}^{\prime} \otimes \mathrm{id}_{M_{11}}\right) \xi_{3}\left(c_{,}\right), c_{,}\right) \\
& =\sqrt{\frac{\operatorname{dim} A_{00} \operatorname{dim} D_{10}}{\operatorname{dim} B_{01} \operatorname{dim} C_{11}}} \operatorname{dim} C_{11} \bar{W}
\end{aligned}
$$

where $\xi_{4}^{\prime} d_{t}=\lim _{n} \sum_{t h} a_{t, k}^{(n)} \otimes z_{t, h}^{(n)}$, with $a_{t, k}^{(n)} \in{ }_{00} A_{10}$ and $z_{l, k}^{(n)} \in{ }_{00}\left(M_{10}\right)_{10}$. This proves the desired equality.

The anti-isomorphism between $M_{00}^{\prime} \cap M_{11}$ and $M_{11}^{\prime} \cap M_{22}$ and the conjugation of the intertwiners give the Initialization axiom. We can choose $c=1$.

The next axiom is flatness. For ordinary paragroups, this axiom follows from commutativity of the tensor product operations from the left and from the right as in [38]. The same proof works in our setting.

The next axiom is the new one, the Intertwining Yang-Baxter Equation. In the formula of the Axiom, the both hand sides are equal to the inner product of the two intertwiners $\xi_{3} \cdot \xi_{2} \cdot \xi_{1}$ and $\xi_{4} \cdot \xi_{5} \cdot \xi_{6}$, so we get this equality. (When making the compositions, we dropped " $\otimes$ id" for simplicity.)

If the subfactor $M_{00} \cap M_{11}$ is of finite depth, the above is enough, but in general, we still need two more axioms. 
We can now make a triple sequence of the string algebras $A_{j k l}$ as in Section 2 and the sequence is identified with the sequences of the higher relative commutants $\left(M_{-J,-\jmath}^{\prime} \cap M_{k l}\right)$. We need one lemma.

Lemma 3.3. Under Assumption 1.1, we choose a generating tunnel

$$
\cdots \subset M_{-2,-2} \subset M_{-1,-1} \subset M_{00} \subset M_{11}
$$

for the subfactor $M_{00} \subset M_{11}$. Then $\vee_{k}\left(M_{-k,-k}^{\prime} \cap M_{l \jmath}\right)=M_{l j}$ for $i, j \geq 0$.

Proof. If $i=j$, we get the conclusion with the definition of the generating tunnel. Let $i=0, j=1$. In the following commuting square,

$$
\begin{array}{ccc}
\vee_{\jmath}\left(M_{-\jmath,-\jmath}^{\prime} \cap M_{01}\right) & \subset M_{01} \\
\cap & & \cap \\
\vee_{\jmath}\left(M_{-\jmath,-\jmath}^{\prime} \cap M_{11}\right) & \subset & M_{11}
\end{array}
$$

we have $\vee_{J}\left(M_{-J,-\jmath}^{\prime} \cap M_{11}\right)=M_{11}$, which implies $\vee_{J}\left(M_{-J,-\jmath}^{\prime} \cap M_{01}\right)=M_{01}$. The other cases are proved similarly.

Q.E.D.

Now we discuss the two remaining axioms. We now have the flat connection $W$ with the intertwining Yang-Baxter equation, so we can make a triple sequence $\left\{A_{j k l}\right\}$ of string algebras as in Section 2 and this system is isomorphic to $\left\{M_{-\jmath,-\jmath}^{\prime} \cap M_{k l}\right\}$. By the above lemma, $\vee_{J} A_{j k l}$ is a factor, which implies the Ergodicity Axiom.

Next we check the Amenability Axiom. With the above identification of the two systems of $\left\{A_{j k l}\right\}$ and $\left\{M_{-J .-\jmath}^{\prime} \cap M_{k l}\right\}$, our $p_{J} \in M_{-(J+1),-(J+1)}^{\prime} \cap M_{\infty, \infty}$ is the Jones projection for the subfactor $M_{-(J+1),(J+1)} \subset M_{-\jmath,-J}$ by the generating property. This implies the desired identities.

Thus we have constructed the system satisfying Axioms $1-9$. We call the system the standard invariant of the commuting square. Note that the algebraic axioms do not need the AFD condition of the type $\mathrm{II}_{1}$ factors.

We now give the converse construction. Next suppose we have a combinatorial system $\left(\mathscr{G}, \tau, \mu, \imath, \beta_{1}, \beta_{2}, W\right)$ satisfying the axioms in Section 2 . We will prove that this indeed comes from a commuting square of type $\mathrm{II}_{1}$ factors satisfying Assumption 1.1.

From a given $\left(\mathscr{G}, \tau, \mu, \imath, \beta_{1}, \beta_{2}, W\right)$, we construct the triple sequence $\left\{A_{\jmath k l}\right\}$ of the string algebras as in Section 1 , and define $M_{k l}=A_{\infty, k, l}$ for all $k, l \in \mathbb{Z}$. By the Ergodicity Axiom, these are AFD type $\mathrm{II}_{1}$ factors. By the commuting square condition coming from the Renormalization axiom, we know that the square 


$$
\begin{array}{ccc}
M_{00} & \subset M_{01} \\
\cap & & \cap \\
M_{10} & \subset M_{11}
\end{array}
$$

satisfies the first two conditions of Assumption 1.1. Then by [41, Proposition 1.2 $2^{\circ}$ ], we know that the double sequence $\left\{M_{k l}\right\}_{k, l \geq 0}$ are obtained by the basic construction from the above commuting square as at the beginning of this section and that

$$
\cdots \subset M_{-2,-2} \subset M_{-1,-1} \subset M_{00} \subset M_{11}
$$

is a tunnel. Note that $E_{A_{\infty}^{\prime}-1-1} \cap A_{\infty \infty \infty \infty}\left(p_{J}\right)=\beta_{1}^{-2} \beta_{2}^{-2}$ for $j \geq 1$ by the Amenability Axiom.

We will prove that $A_{j k l}=M_{-\jmath,-\jmath}^{\prime} \cap M_{k l}$ for $j, k, l \geq 0$ by an argument of [37, page 35]. Note that elements in $A_{\infty,-n,-n}$ and $A_{n, \infty, \infty}$ commute for $n \geq 0$ by the Flatness Axiom and [24, Theorem 2.1]. Without loss of generality, we may assume $j=0$ and $k=l$. Take $x \in A_{\infty, 0,0}^{\prime} \cap A_{\infty, h, k}$ and set $x_{n}=E_{A_{n k h}}(x) \in A_{n 00}^{\prime} \cap A_{n k k}$ for $n \geq 0$. Then $x_{n} \in A_{n, \infty, \infty}$ is written as a finite sum $\sum_{l} a_{l} p_{n-1} b_{l}$, where $a_{l}, b_{l} \in A_{n-1, \infty, \infty}$. Then

$$
\begin{aligned}
\left\|x-x_{n}\right\|_{2} & \geq\left\|E_{A_{\infty}^{\prime}-(n-1),-n-1, \cap A_{\infty \infty \infty}}\left(x-x_{n}\right)\right\|_{2} \\
& =\left\|x-\sum_{1} a_{t} E_{A_{\infty}^{\prime}-(n-1)-(n-1)} \cap A_{\infty \infty \infty}\left(p_{n-1}\right) b_{l}\right\|_{2} \\
& =\left\|x-\beta_{1}^{-2} \beta_{2}^{-2} \sum_{l} a_{l} b_{l}\right\|_{2} \\
& =\left\|x-E_{A_{n-1} \infty \infty}\left(x_{n}\right)\right\|_{2} \\
& =\left\|x-x_{n-1}\right\|_{2}
\end{aligned}
$$

Because $\lim _{n}\left\|x-x_{n}\right\|_{2}=0$, we get $\left\|x-x_{n}\right\|_{2}=0$ for all $n \geq 0$, and in particular $x=x_{0} \in A_{0, k, k}$.

Then $e_{1} f_{1} \in A_{022}$ is the Jones projection for the subfactor $M_{00} \subset M_{11}$, and it is also the Jones projection for the subfactor $A_{-2, \infty, \infty} \subset A_{-1, \infty, \infty}$ by the identification based on the Initialization Axiom. (Note that $A_{\jmath, \infty, \infty}$ is a factor by the Ergodicity Axiom.) We now get $E_{M_{11}^{\prime} \cap M_{22}}\left(e_{1} f_{1}\right)=E_{A_{-122}}\left(e_{1} f_{1}\right)=E_{A_{-1 \infty \infty}}\left(e_{1} f_{1}\right)=\beta_{1}^{-2} \beta_{2}^{-2}$, thus $M_{11} \subset M_{22}$ is extremal. By [44, Theorem 5.3.1 (ii)], we know that the subfactor $M_{00} \subset M_{11}$ is strongly amenable and the square

$$
\begin{array}{ccc}
M_{00} & \subset M_{01} \\
\cap & & \cap \\
M_{10} & \subset M_{11}
\end{array}
$$


satisfies all the conditions of Assumption 1.1. Now $M_{-J,-\jmath}^{\prime} \cap M_{k l}$ is given by $A_{j k l}$, thus we get back the original system $\left(\mathscr{G}, \tau, \mu, \imath, \beta_{1}, \beta_{2}, W\right)$ by the above procedure in this Section.

We have thus proved the following theorem.

Theorem 3.4 (Main Theorem)。There is a bijective correspondence between isomorphism classes of commuting squares of type $\mathrm{II}_{1}$ factors satisfying Assumption 1.1 and isomorphism classes of combinatorial systems $\left(\mathscr{G}, \tau, \mu, \imath, \beta_{1}, \beta_{2}, W\right)$ satisfying Axioms $1-9$ in Section 1.

Roughly speaking, our classification deals with the objects which is onedimensional higher than ordinary paragroups for classification of subfactors. Thus we have the following correspondence table.

\begin{tabular}{|c|c|}
\hline Subfactor & Paragroup action on subfactor \\
\hline (dual) principal graph & (dual) principal connection \\
\hline (canonical) Commuting square & (canonical) commuting cube \\
\hline double sequence of string algebras & triple sequence of string algebras \\
\hline
\end{tabular}

Table 1 .

With this theorem, we can give a solution to Okamoto's problem which was mentioned in the Introduction. Of course, one way to get a characterization is to compare our standard invariant and that of the commuting square arising as a tensor product of two subfactors, but it is not easy to see what kind of conditions we have as a characterization in this way. Another way of writing down a characterization is as follows.

Proposition 3.5. Suppose that the commuting square

$$
\begin{array}{ccc}
M_{00} & \subset M_{01} \\
\cap & & \cap \\
M_{10} & \subset M_{11}
\end{array}
$$

satisfies Assumption 1.1. This commuting square is isomorphic to the following type of commuting square

$$
\begin{array}{ccc}
N \otimes P & \subset & M \otimes P \\
\cap & & \cap \\
N \otimes Q & \subset & M \otimes Q
\end{array}
$$

if and only if the following three conditions are satisfied. 
1. $M_{00}^{\prime} \cap M_{0_{J}} \subset M_{10}^{\prime} \cap M_{0_{J}}$ for all $j \geq 0$.

2. $M_{00}^{\prime} \cap M_{j 0} \subset M_{01}^{\prime} \cap M_{\text {J0 }}$ for all $j \geq 0$.

3. $M_{00}^{\prime} \cap M_{j k}=\left(M_{00}^{\prime} \cap M_{0 k}\right) \otimes\left(M_{00}^{\prime} \cap M_{j 0}\right)$ for all $j, k \geq 0$.

Proof. By Condition 1, we get $M_{00}^{\prime} \cap M_{0 J} \subset M_{n, 0}^{\prime} \cap M_{n, J}$ for all even $n \geq 0$, and by comparing the dimension of the both hand sides, we have the equality. Similarly, we get $M_{00}^{\prime} \cap M_{j 0} \subset M_{0,2 n}^{\prime} \cap M_{\jmath, 2 n}$ for all even $n \geq 0$. Choose a generating tunnel

$$
\cdots \subset M_{-2,-2} \subset M_{-1,-1} \subset M_{00} \subset M_{11} .
$$

Then by Condition 3, the square

$$
\begin{array}{ccc}
M_{-n,-n}^{\prime} \cap M_{00} & \subset & M_{-n,-n}^{\prime} \cap M_{01} \\
\cap & & \cap \\
M_{-n,-n}^{\prime} \cap M_{10} & \subset & M_{-n,-n}^{\prime} \cap M_{11}
\end{array}
$$

is identical with

$$
\begin{aligned}
& \left(M_{-n,-n}^{\prime} \cap M_{-n, 0}\right) \otimes\left(M_{-n,-n}^{\prime} \cap M_{0,-n}\right) \subset\left(M_{-n,-n}^{\prime} \cap M_{-n, 1}\right) \otimes\left(M_{-n,-n}^{\prime} \cap M_{0,-n}\right) \\
& \left(M_{-n,-n}^{\prime} \cap M_{-n, 0}\right) \otimes\left(M_{-n,-n}^{\prime} \cap M_{1,-n}\right) \subset\left(M_{-n,-n}^{\prime} \cap M_{-n, 1}\right) \otimes\left(M_{-n,-n}^{\prime} \cap M_{1,-n}\right)
\end{aligned}
$$

and by the above, this is identical with

$$
\begin{array}{ccc}
\left(M_{0,-n}^{\prime} \cap M_{0,0}\right) \otimes\left(M_{-n, 0}^{\prime} \cap M_{0,0}\right) & \subset\left(M_{0,-n}^{\prime} \cap M_{0,1}\right) \otimes\left(M_{-n, 0}^{\prime} \cap M_{0,0}\right) \\
\cap & \cap \\
\left(M_{0,-n}^{\prime} \cap M_{0,0}\right) \otimes\left(M_{-n, 0}^{\prime} \cap M_{1,0}\right) & \subset\left(M_{0,-n}^{\prime} \cap M_{0,1}\right) \otimes\left(M_{-n, 0}^{\prime} \cap M_{1,0}\right)
\end{array}
$$

for even $n \geq 0$.

Because $M_{00} \subset M_{01}$ and $M_{00} \subset M_{10}$ are strongly amenable by [44], we get the conclusion.

The "only if" part is trivial.

Q.E.D.

Check the above conditions in the case of the following commuting square.

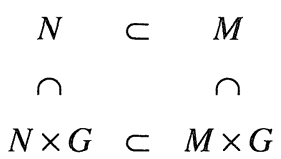

where $G$ is a finite group giving outer actions on $M, N$. Then we reach an "interpretation" of the above conditions as follows. The first two conditions are regarded as an "algebraic analogue" of approximate innerness of the paragroup action. (More precisely, these conditions are "triviality of the Loi invariant for paragroup actions", as understood from the special type of commuting squares 
like (1). One of these two does not imply the other, so we need two conditions.) The third condition corresponds to the "strong outerness" of the paragroup action, which is an algebraic counterpart of central freeness. If the commuting square of. type $\mathrm{II}_{1}$ factors is like (1), then it is enough to check the third condition for $j=1$, because of the vertical depth is 2 . Also note that the condition $M_{00}^{\prime} \cap M_{11}=\mathbb{C}$ in [51] is also a kind of strong outerness because both $M_{00} \subset M_{01}$ and $M_{00} \subset M_{10}$ have depth 2 in [51]. Thus we can interprer the above theorem as saying that "centrally free and approximately inner paragroup action splits", which is an analogue of a group action classification in [34]. (Also see [31], [45], [46].) A more detailed comparison with [46] would be interesting.

We got a characterization in the above form, but this is not so interesting because the above conditions, especially Condition 3, are very strong, and this kind of commuting squares are rather trivial, so we can ask a question: Do we really have many examples of commuting squares of type $I_{1}$ factors satisfying Assumption 1.1 and having non-integer indices which are not of the tensor product type? In the following sections, we will give many such examples and discuss their relations to RCFT and TQFT.

\section{§4. Rational Conformal Field Theory and Paragroup Actions on Subfactors}

In [6], de Boer-Goeree constructed a paragroup from a Rational Conformal Field Theory in the sense of Moore-Seiberg [32]. In particular, if we start with a Wess-Zumino-Witten model with a level $k$, we get a corresponding paragroup. For example, the subfactors of Jones of type $A_{n}$ [21] and those of Wenzl [58] are now obtained as such subfactors corresponding to $S U(N)$. Flatness is the most important axiom for paragroups, and the proof of flatness in [6, Section 4] is based on regular isotopy invariance of partition functions associated to knotted graphs, but if one looks at the proof carefully, one notices that it does not use the Yang-Baxter equation, i.e., invariance under Reidemeister move III. We show that we can construct a system $\left(\mathscr{G}, \tau, \mu, \imath, \beta_{1}, \beta_{2}, W\right)$ satisfying Axioms 1-9 from an RCFT with essential use of the Yang-Baxter equation. In some "good" cases, the Yang-Baxter equation implies flatness as pointed out in [10], but in general neither of the Yang-Baxter equation and the flatness implies the other. So our construction here shows the missing role of the Yang-Baxter equation in paragroup/subfactor theory and this can be regarded as a result of a principle that "RCFT has a higher symmetry than ordinary paragroups".

We proceed so that our construction generalizes that in [6, Section 4]. Fix an RCFT. Our notations are slightly different from those in [6] and compatible with those in [63]. Choose fields $x, y$ from the RCFT. Tentatively, we set all the eight ${ }_{i j} \mathscr{G}_{k l}$ 's to be the set of the primary fields in the RCFT. For 
$a \in{ }_{00} \mathscr{G}_{00}$ and $b \in{ }_{00} \mathscr{G}_{01}$, the number of edges in ${ }_{00}^{00} \mathscr{G}_{01}^{00}$ connecting $a, b$ is given by $N_{x a}^{b}$, which is the multiplicity of $b$ in $x a$. Similarly, for the graphs ${ }_{11}^{11} \mathscr{G}_{01}^{00},{ }_{00}^{00} \mathscr{G}_{11}^{10},{ }_{10}^{11} \mathscr{G}_{11}^{11},{ }_{00}^{00} \mathscr{G}_{10}^{00},{ }_{11}^{11} \mathscr{G}_{10}^{00},{ }_{00}^{00} \mathscr{G}_{11}^{01},{ }_{11}^{11} \mathscr{G}_{11}^{01},{ }_{11}^{00} \mathscr{G}_{00}^{00},{ }_{11}^{00} \mathscr{G}_{01}^{01},{ }_{11}^{00} \mathscr{G}_{10}^{10}$, and ${ }_{11}^{00} \mathscr{G}_{11}^{11}$, we use the multiplicities $N_{x a}^{b}, N_{\star a}^{b}, N_{x a}^{b}, N_{v a}^{b}, N_{v a}^{b}, N_{v a}^{b}, N_{\imath a}^{b}, \sum_{c} N_{v c}^{b} N_{\lambda a}^{c}, \sum_{c} N_{v c}^{b} N_{\lambda a}^{c}$, $\sum_{c} N_{y c}^{b} N_{x a}^{c}$, and $\sum_{c} N_{y c}^{b} N_{\imath a}^{c}$ respectively. Let $\mathscr{G}$ be the connected component containing $1 \in_{00} \mathscr{G}_{00}$ of the resulting graph. We set $\beta_{1}=S_{0 x} / S_{00}$, and $\beta_{2}=S_{0 y} / S_{00}$. We set $*_{00}=1 \in_{00} \mathscr{G}_{00}$ and $*_{11}=1 \in_{11} \mathscr{G}_{11}$. The contragredient map is defined by $\tau(a)=a^{\vee}$.

We define the connection as follows. If $a \in_{00} \mathscr{G}_{00}, b \in_{00} \mathscr{G}_{01}, c \in_{00} \mathscr{G}_{11}, d \in_{00} \mathscr{G}_{10}$, or $a \in_{11} \mathscr{G}_{00}, b \in_{11} \mathscr{G}_{01}, c \in_{11} \mathscr{G}_{11}, d \in_{11} \mathscr{G}_{10}$, then we set as in Figure 8.

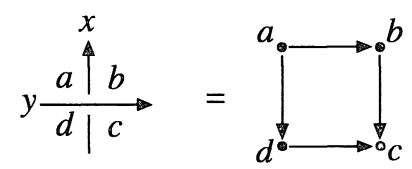

Figure 8 .

Here if any of $N_{a x}^{b}, N_{d x}^{c}, N_{a y}^{d}, N_{b y}^{c}$ is bigger than 1, we actually need labeling for the edges, but we omit the labeling for simplicity as in [6]. Similary, if $a \in{ }_{00} \mathscr{G}_{00}, b \in{ }_{00} \mathscr{G}_{01}, c \in{ }_{11} \mathscr{G}_{01}, d \in \in_{11} \mathscr{G}_{00}$, or $a \in_{00} \mathscr{G}_{10}, b \in_{00} \mathscr{G}_{11}, c \in_{11} \mathscr{G}_{11}, d \in_{11} \mathscr{G}_{10}$, then we set as in Figure 9.

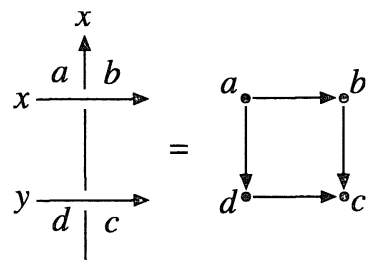

Figure 9.

If $a \in_{00} \mathscr{G}_{00}, b \in_{11} \mathscr{G}_{00}, c \in_{11} \mathscr{G}_{10}, d \in_{00} \mathscr{G}_{10}$, or $a \in_{00} \mathscr{G}_{01}, b \in_{11} \mathscr{G}_{01}, c \in_{11} \mathscr{G}_{11}, d \in_{00} \mathscr{G}_{11}$, then we set as in Figure 10.

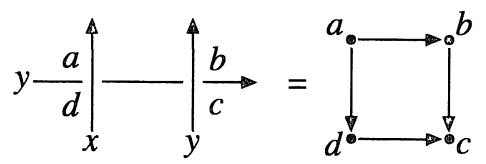

Figure 10

Then we set the other values of the connection so that the Renormalization Axiom holds. (Note that our convention of normalizing constants is different from that of [35], so we do not need the coefficient of 4th roots which they have in [6] for the definition of the connection.) We get the Unitarity Axiom by the unitarity of 
braiding matrix as in [6]. The Initialization Axiom follows from natural identification of edges, and flatness follows from the same argument as in [6] based on the regular isotopy invariance of the partition functions associated to knotted graphs. The Intertwining Yang-Baxter Equation follows from Figure 11.
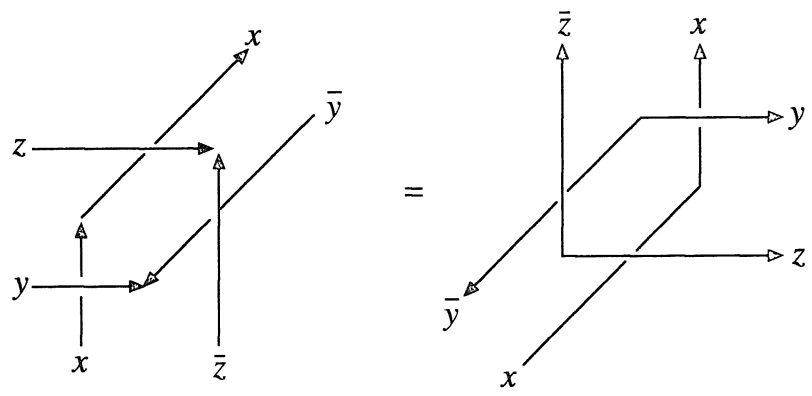

Figure 11. The Yang-Baxter equation

Here we set $z=x, y$. Because the graph $\mathscr{G}$ is finite, the other two axioms hold automatically. This system is far from being that of a tensor product of two subfactors.

As a very simple example of this construction, take a Lie group $S U(2)$ and set level $k$ to be $n-1$. In this case, we have a flat connection on the graphs $A_{n}$ as in [35], and it is well-known that it satisfies the Yang-Baxter equation. (It follows from the flatness of the Jones projections. Also compare the formula with the one in [2].) So we can construct an action of a paragroup of type $A_{n}$ on a subfactor of type $A_{n}$. It is easy to identify this commuting square with the following. Take a subfactor $N \subset M$ of type $A_{n}$, and make a basic construction $N \subset M \subset M_{1}$. Set $\varepsilon=\sqrt{-1} \exp (\pi \sqrt{-1}) / 2(n+1), \quad \beta=2 \cos (\pi /(n+1))$, and $u=\varepsilon+\bar{\varepsilon} \beta e$, then $u$ is a unitary, and we get a commuting square

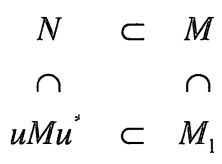

(Also see [16, Example 4.2.10].)

Furthermore, the orbifold construction [10], [24] has been clarified by $\mathrm{Xu}$ [63], [64] in the settings of RCFT. It is easy to see that the same construction works in our settings here. That is, if the obstruction for flatness arising from a conformal dimension is trivial as in [63, formulas (2), (3)], then the orbifold construction with an RCFT gives a system satisfying our Axioms $1-9$. This is because the Yang-Baxter equation is preserved in the orbifold construction as in [10]. The axioms except for the flatness and the Intertwining Yang-Baxter Equations are automatically satisfied as in [10]. 


\section{§5. Topological Quatum Field Theory for the Goodman-de la Harpe-Jones Subfactors}

In this section, we look for another paragroup action which does not come form RCFT and discuss the construction of subfactors by Goodman-de la HarpeJones [16] based on the Dynkin diagrams. Goodman-de la Harpe-Jones constructed some new subfactors in [16, Section 4.5], and their subfactor with index $3+\sqrt{3}$ has caught special attentions, because it is one of the few known subfactors with non-integer indices which do not come from Wess-ZuminoWitten models and it has relatively small index. (Note that most of the known subfactors come from Wess-Zumino-Witten models, their orbifolds, and group/Hopf algebra actions.) Okamoto computed its principal graph and showed that the subfactor has finite depth in [39]. (Also see [25, Remark 2.2].) From the commuting squares in [16, Section 4.5], we have a bi-unitary connection [53], [36], and the flatness of the Jones projections implies the Intertwining YangBaxter Equation with the formula $\left(S^{\prime}\right)$ in [50, page 405], as noted by Jones. So we can make a triple sequence of the string algebras as in Section 2 from this connection. In the case of $E_{6}$ and $A_{11}$, our graph, which gives the Bratteli diagrams of the commuting cube, looks like Figure 12.

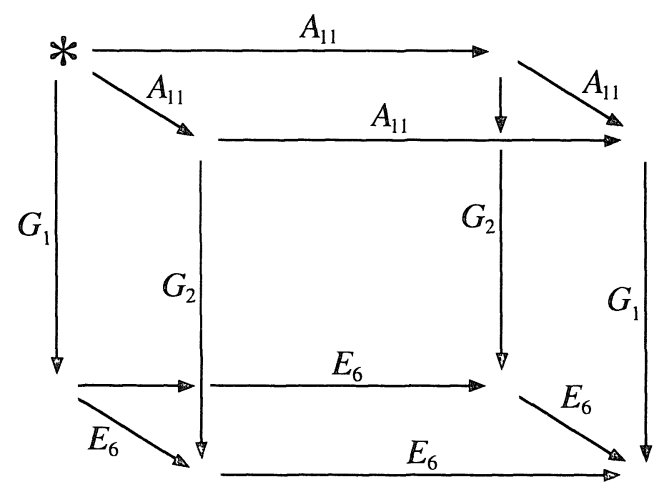

Figure 12.

Here the graphs $G_{1}, G_{2}$ are as in Figure 13.
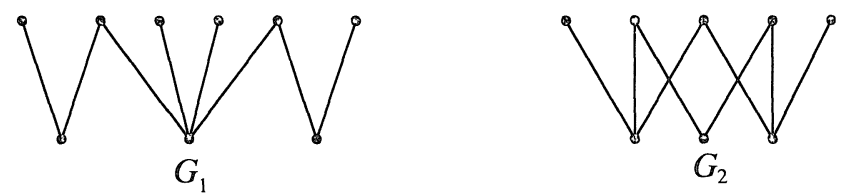

Figure 13.

Note that for the top face of the cube in Figure 12, we use the standard 
connection for $A_{11}$, for the bottom face of the cube, we use the standard connection for $E_{6}$, and for the four side faces, we use the connection appearing in the Goodman-de la Harpe-Jones commuting square [16]. The explicit formula for the last connection is given in the table in [50, page 418]. (Roche verified the intertwining Yang-Baxter Equation by direct computation, but it is a consequence of the flatness of the Jones projections as noted above.) From the triple sequence of the string algebras, we get a commuting square

$$
\begin{array}{ccc}
M_{00} & \subset & M_{01} \\
\cap & & \cap \\
M_{10} & \subset M_{11}
\end{array}
$$

satisfying Assumption 1.1. Here the subfactor $M_{00} \subset M_{01}$ is of type $A_{11}$, $M_{10} \subset M_{11}$ is of type $E_{6}$, and $M_{00} \subset M_{10}$ and $M_{01} \subset M_{11}$ are the Goodman-de la Harpe-Jones subfactor with index $3+\sqrt{3}$. (There are two flat connections on $E_{6}$, so we have two commuting squares as (2) corresponding to the two connections.) The flatness of the Jones projection implies flatness with respect to the $*$, which is shared by $A_{11}, E_{6}$ and $G_{1}$, as in [25, Remark 2.2], so we conclude that the original connection arising from the Goodman-de la Harpe-Jones commuting square is the "principal connection" which is a part of our standard invariant. The above graph $G_{2}$ of Figure 13 cannot be a principal graph of any subfactor because it is rejected by the " $2 \cos (\pi / n)$-rule" [18, Theorem 6.1], but it does not appear as a part of the standard invariant of a paragroup action on a subfactor. Similar results holds for the case $E_{7}$ and $E_{8}$, for which [9] makes several computations.

We can compute the fusion rule of the $N-N$ bimodules of the Goodman-de la Harpe-Jones subfactor $N \subset M$ with index $3+\sqrt{3}$ with this observation on the principal connection. The flatness of the Jones projections implies that all the irreducible summands of ${ }_{00}\left(M_{k l}\right)_{00}$ appear in the irreducible decomposition of ${ }_{00}\left(M_{0 l}\right)_{00}$ for large $l$. Thus $G_{1}={ }_{00}^{00} \mathscr{G}_{00}^{10}$ and the even vertices of the $A_{11}$ and those of $G_{1}$ are identified, and this identification means that these two systems of bimodules are the same. (It is also possible to give more direct identification of these systems of bimodules based on Ocneanu's parallel transport [35].) That is, the fusion rule of the $N-N$ bimodules for our subfactor is the same as that of the subfactor of type $A_{11}$. With the labeling of even vertices as in Figure 14,

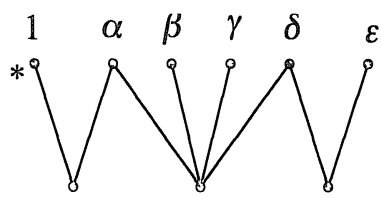

Figure 14 .

we get the following multiplication Table 2 . 


\begin{tabular}{|c|c|c|c|c|c|}
\hline$\times$ & $\alpha$ & $\beta$ & $\gamma$ & $\delta$ & $\varepsilon$ \\
\hline$\alpha$ & $1+\alpha+\beta+\gamma+\delta$ & $\alpha+\gamma+\delta$ & $\alpha+\beta+\delta$ & $\alpha+\beta+\gamma+\delta+\varepsilon$ & $\delta$ \\
\hline$\beta$ & $\alpha+\gamma+\delta$ & $1+\beta+\delta$ & $\alpha+\gamma+\varepsilon$ & $\alpha+\beta+\delta$ & $\gamma$ \\
\hline$\gamma$ & $\alpha+\beta+\delta$ & $\alpha+\gamma+\varepsilon$ & $1+\beta+\delta$ & $\alpha+\gamma+\delta$ & $\beta$ \\
\hline$\delta$ & $\alpha+\beta+\gamma+\delta+\varepsilon$ & $\alpha+\beta+\delta$ & $\alpha+\gamma+\delta$ & $1+\alpha+\beta+\gamma+\delta$ & $\alpha$ \\
\hline$\varepsilon$ & $\delta$ & $\gamma$ & $\beta$ & $\alpha$ & 1 \\
\hline
\end{tabular}

Table 2. Multiplications of the $N-N$ bimodules

D. Bisch [4] tried to compute this fusion rule just from the principal graph, but he had five possibilities, and could not determine the right one. Our computation shows that the fifth table in [4] is the correct table for the $N-N$ bimodules.

Our commuting cube, however, does not satisfy the Initialization Axiom, so we cannot compute the "dual principal connection" directly, which is the same situation as the method in [39] did not give the dual principal graphs. Our next aim is to compute the Bratteli diagram for this "dual principal connection".

First as pointed out in [25] (and independently noted by U. Haagerup), the dual principal graph of the Goodman-de la Harpe-Jones subfactor $N \subset M$ with index $3+\sqrt{3}$ is the same as the principal graph $G_{1}$. Because the proof was not presented in [25], we give a sketch here. The Bratteli diagram for the higher relative commutants $N^{\prime} \cap M_{k}$ is as in Figure 15.

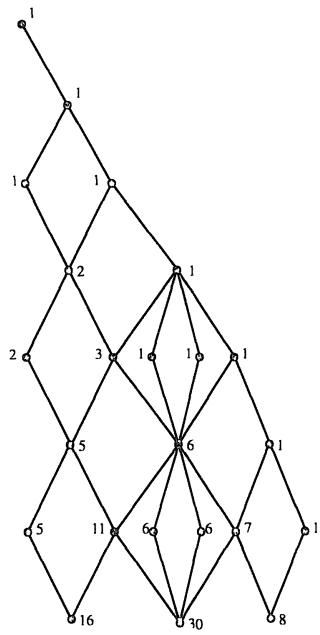

Figure 15. The Bratteli diagram for $N^{\prime} \cap M_{k}$

Then the odd levels of this tower must coincide with the odd levels of the other higher relative commutants $M^{\prime} \cap M_{k}$, because they are identified with the antiisomorphisms $J \cdot{ }^{\prime} J$. This fact forces for the dual principal graph to be the same 
as principal graph.

We look for the graphs ${ }_{11}^{11} \geqslant 00,11 \geqslant, 11,11 \%, 01$, and ${ }_{11}^{11} \% 00$. The above observation shows that a connected component of the graph ${ }_{10}^{11} \mathscr{G}_{11}^{11}$ is $E_{6}$, and a connected component of the graph ${ }_{11}^{11} \mathscr{G}_{11}^{01}$ is $G_{1}$. Because the number of vertices in ${ }_{00} \mathscr{G}_{11}$ is three, the number of vertices in ${ }_{11} \mathscr{G}_{00}$ is also three and two of them have the same weight $\mu(\cdot)$ by the contragradient map. Any connected component of the graph ${ }_{11}^{11} \mathscr{G}_{01}^{00}$ has the Perron-Frobenius eigenvalue $2 \cos (\pi / 12)$, so each connected component must be one of $A_{11}, D_{7}$, and $E_{6}$. The above observation on ${ }_{11} \mathscr{G}_{00}$ shows that ${ }_{11}^{11} \mathscr{G}_{01}^{00}$ is connected and equal to $E_{6}$, and it implies that ${ }_{11}^{11} \mathscr{G}_{11}^{01}$ is connected and equal to $G_{1}$. It then implies that ${ }_{10}^{11} \mathscr{G}_{11}^{11}$ has two connected components and the other is another copy of $E_{6}$, and this uniquely determines ${ }_{11}^{11} \mathscr{G}_{01}^{00}$. The graph we got are as in Figure 16.

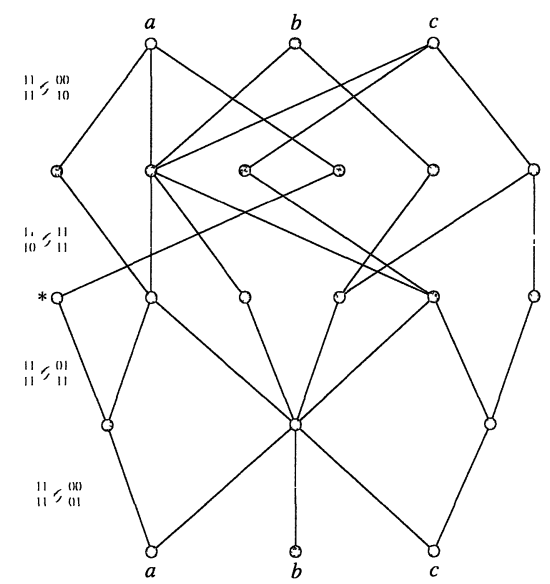

Figure 16. The Bratteli diagrams for the dual principal connection

This is a non-trivial example where a disconnected graph appears as a part of the standard invariant. It shows that the three even vertices of $E_{6}$ are identified with three of the six even vertices of $\mathscr{G}_{1}$ which is now the dual principal graph of $N \subset M$. This shows that the fusion algebra of the $M-M$ bimodules for our subfactor $N \subset M$ contains a sub-fusion algebra which is isomorphic to the fusion algebra of even vertices of $E_{6}$, which was computed in [18]. This determines the fusion algebra of $M-M$ bimodules. With the same labeling of even vertices of $G_{1}$ as in Figure 14, we get the following multiplication Table 3.

\begin{tabular}{|c|c|c|c|c|c|}
\hline$\times$ & $\alpha$ & $\beta$ & $\gamma$ & $\delta$ & $\varepsilon$ \\
\hline$\alpha$ & $1+\alpha+\beta+\gamma+\delta$ & $\alpha+\gamma+\delta$ & $\alpha+\beta+\delta$ & $\alpha+\beta+\gamma+\delta+\varepsilon$ & $\delta$ \\
\hline$\beta$ & $\alpha+\gamma+\delta$ & $1+2 \beta+\varepsilon$ & $\alpha+\delta$ & $\alpha+\gamma+\delta$ & $\beta$ \\
\hline$\gamma$ & $\alpha+\beta+\delta$ & $\alpha+\delta$ & $1+2 \gamma+\varepsilon$ & $\alpha+\beta+\delta$ & $\gamma$ \\
\hline$\delta$ & $\alpha+\beta+\gamma+\delta+\varepsilon$ & $\alpha+\gamma+\delta$ & $\alpha+\beta+\delta$ & $1+\alpha+\beta+\gamma+\delta$ & $\alpha$ \\
\hline$\varepsilon$ & $\delta$ & $\beta$ & $\gamma$ & $\alpha$ & 1 \\
\hline
\end{tabular}

Table 3. Multiplications of the $M-M$ bimodules 
This is the first table among the five in [4]. This means that the fusion algebras for $N-N$ bimodules and $M-M$ bimodules of the Goodman-de la Harpe-Jones subfactor $N \subset M$ with index $3+\sqrt{3}$ are different, though the principal graph and the dual principal graphs are the same. This subfactor is the first example of such kind. A recent work of U. Haagerup [17] shows that this example has the smallest index among such subfactors. (Note that different finite groups with the same order give different fusion algebras on the same principal graphs, but these two cannot be a principal graph and a dual principal graph of one subfactor.) In particular, the subfactor $N \subset M$ is not conjugate to its dual $M \subset M_{1}$. (M. Izumi commuted the flat connection of this subfactor, and it also follows from his computation that $N \subset M$ and $M \subset M_{1}$ are not conjugate. It, however, seems difficult to see the difference of the fusion rules from the flat connection.).

Furthermore, a 3-dimensional topological quantum field theory (TQFT) of Turaev-Viro type [55] based of triangulations arising from subfactors [38], [11] is computed with $6 j$-symbols of only $N-N$ bimodules (or $M$ - $M$ bimodules). So the TQFT for the Goodman-de la Harpe-Jones subfactor with index $3+\sqrt{3}$ is the same as that for the subfactor of type $A_{11}$. Similar results holds for the subfactors in [16] arising from the Dynkin diagrams $E_{7}$ and $E_{8}$.

The TQFT's arising from subfactors of type $E_{6}$ use the sub-fusion algebras of the fusion algebra of the $M-M$ bimodules of the Goodman-de la Harpe-Jones subfactor with index $3+\sqrt{3}$ which gives the same TQFT as the $N-N$ bimodules of the same subfactor and as the $A_{11}$ TQFT. In this sense, the $E_{6}$ TQFT's use just a partial information of the $A_{11} \mathrm{TQFT}$, but it does not necessarily mean that the $E_{6}$ TQFT's are less interesting. Indeed, Ocneanu said to the author that the $E_{6}$ TQFT's do detect orientations of certain lens spaces by a computation of Niţică and Török [33], while it is easy to see that the $A_{11}$ TQFT does not detect an orientation of any 3-manifold. Also note that there are two subfactors of type $E_{6}$, and they correspond to two vertices $\beta, \gamma$ of the dual principal graph of the Goodman-de la Harpe-Jones subfactor.

If we make the same construction for the $D_{n}$ diagrams, we again have a graph $D_{n}$ as a part of the standard invariant. The graphs $D_{2 n+1}$ are impossible as a principal graph [35], [18], [24], [54], but it does appear as a part of the standard invariant of a paragroup action on a subfactor.

Furthermore, we obtained the following type of commuting square in the computation of the flat part of the non-flat connections on $E_{7}$ [12]. (See [13] for relations with CFT.)

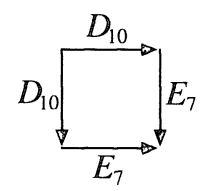

We again have the Intertwining Yang-Baxter Equation from the flatness of the 
Jones projection, thus we can repeat the construction of the triple sequence of string algebras. Then the computation in [12] shows that the above connection is really a "principal connection" of our commuting square of type $\mathbb{I I}_{1}$ factors. This shows that the graph $E_{7}$ also appear as a part of the standard invariant of a paragroup action on a subfactor.

\section{§6. Coset Comstruction for Subfactors}

Finally we discuss an analogue of the coset construction of RCFT in a subfactor theory. In [6, Section 10], de Boer-Goeree has a correspondence table between four constructions in subfactor theory and RCFT. The orbifold construction is one of them, and it has been studied in detail in [10], [13], [24], [25], [26], [63], [64]. The tensor product construction is not so interesting in subfactor theory, and the "extended algebras" is a construction of $N \times G \subset M \times G$ from $N \subset M$. If we want to get a subfactor $N \times G \subset M \times G$ with finite depth from a subfactor $N \subset M$ with finite depth, this is essentially same as the orbifold construction, so it is again not so interesting. The last one of the four is the coset construction, which gives $S^{\prime} \cap N \subset S^{\prime} \cap M$ from a subfactor $N \subset M$ and $S \subset N$.

We regard this as a purely operator algebraic problem to construct a new subfactor $S^{\prime} \cap N \subset S^{\prime} \cap M$ from a given subfactor $N \subset M$ for an appropriate choice of $S \subset N$.

To get factors $S^{\prime} \cap N$ and $S^{\prime} \cap M$, the subalgebra $S$ must be a factor. If $S$ is of finite dimension, there is nothing interesting, so we must assume that $S$ is a subfactor of $N$. If $[N: S]<\infty, S^{\prime} \cap N$ is finite dimensional, so we must assume $[N: S]=0$. Thus our problem can be also stated as follows.

For a given subfactor $N \subset M$, what kind of subfactors $S^{\prime} \cap N \subset S^{\prime} \cap M$ do we get, where $S$ is a subfactor of $N$ with infinite index such that $S^{\prime} \cap N$ and $S^{\prime} \cap M$ are factors? This can be regarded as a classification problem for $S$ (for a fixed $N \subset M)$, too, and then the paragroup of $S^{\prime} \cap N \subset S^{\prime} \cap M$ is an invariant for $S$.

Note that by the commuting square condition, we get $\left[S^{\prime} \cap M: S^{\prime} \cap N\right] \leq[M: N]$, and we expect that natural constructions would give an equality here. (The equality does not hold in general. Take a subfactor $P \subset Q$ with $P^{\prime} \cap Q=\mathbb{C}$, and then set $S=P \otimes \mathbb{C} \subset N=P \otimes P \subset M=Q \otimes P$. Of course, such a construction is not interesting for us.)

First we note the following proposition.

Proposition 6.1. Let $N \subset M$ be a subfactor with finite index and $S$ be a subfactor of $N$ such that $S^{\prime} \cap N$ and $S^{\prime} \cap M$ are type $\mathbb{I}_{1}$ factors with $\left[S^{\prime} \cap M: S^{\prime} \cap N\right] \leq[M: N]$. Then it is possible to choose a tunnel.

$$
\cdots \subset N_{2} \subset N_{1} \subset N \subset M
$$


so that $N_{,} \supset S$ and

$$
\cdots \subset S^{\prime} \cap N_{2} \subset S^{\prime} \cap N_{1} \subset S^{\prime} \cap N \subset S^{\prime} \cap M
$$

is a tunnel for $S^{\prime} \cap N \subset S^{\prime} \cap M$. We have $N_{j}^{\prime} \cap N \subset\left(S^{\prime} \cap N_{J}\right)^{\prime} \cap\left(S^{\prime} \cap N\right)$ and $N_{j}^{\prime} \cap M \subset\left(S^{\prime} \cap N_{J}\right)^{\prime} \cap\left(S^{\prime} \cap M\right)$.

Proof. First choose a projection $e_{0} \in S^{\prime} \cap M$ with $E_{S^{\prime} \cap N}\left(e_{0}\right)=$ $\left[S^{\prime} \cap M: S^{\prime} \cap N\right]^{-1}=[M: N]^{-1}$. By the commuting square condition, $E_{N}\left(e_{0}\right)=$ $[M: N]^{-1}$, so $N_{1}=N \cap\left\{e_{0}\right\}^{\prime}$ gives a downward basic construction $N_{1} \subset N \subset M$. Because $e_{0} \in S^{\prime} \cap M$, we get $N_{1} \supset S$. Because $S^{\prime} \cap N_{1}=\left(S^{\prime} \cap N\right) \cap\left\{e_{0}\right\}^{\prime}$, we also know that $S^{\prime} \cap N_{1} \subset S^{\prime} \cap N \subset S^{\prime} \cap M$ is a downward basic construction. By repeating this argument, we get a desired tunnel. The last two inclusions are then trivial.

Q.E.D.

We call a construction of $S^{\prime} \cap N \subset S^{\prime} \cap M$ from $N \subset M$ as above the coset construction. The above proposition shows that the coset construction does not decrease the higher relative commutants.

The next proposition gives a basis of the coset construction in our settings.

Lemma 6.2. Suppose that the following square satisfies Assumption 1.1

$$
\begin{array}{ccc}
M_{00} & \subset & M_{01} \\
\cap & & \cap \\
M_{10} & \subset & M_{11}
\end{array}
$$

We make a double sequence $\left\{M_{k l}\right\}_{h, l \geq 0}$ of type $\mathrm{II}_{1}$ factors with basic constructions and further assume the condition $M_{00}^{\prime} \cap M_{0 l} \subset M_{10}^{\prime} \cap M_{1 l}$ for $l \geq 0$.

We define $M_{\infty, l}$ as the GNS-completion of $\vee_{k} M_{k l}$ with respect to the trace. Then

$$
M_{\infty, 0} \subset M_{\infty, 1} \subset M_{\infty, 2} \subset M_{\infty, 3} \subset \cdots
$$

is the Jones tower, and we get two equalities $M_{\infty, 0}^{\prime} \cap M_{\infty, l}=M_{00}^{\prime} \cap M_{0 l}$ and $M_{\infty, 1}^{\prime} \cap M_{\infty, l}=M_{01}^{\prime} \cap M_{0 l}$ for $l \geq 1$, which imply that the subfactors $M_{00} \subset M_{01}$ and $M_{\infty, 0} \subset M_{\infty, 1}$ are conjugate.

Proof. The Jones projections for the towers

$$
M_{k 0} \subset M_{k 1} \subset M_{k 2} \subset M_{k 3} \subset \cdots
$$

are common for all $k$. From this fact and the commuting square condition, it is easy to see that

$$
M_{\infty, 0} \subset M_{\infty, 1} \subset M_{\infty, 2} \subset M_{\infty, 3} \subset \cdots
$$

is the Jones tower. Any element in $M_{00}^{\prime} \cap M_{0 l}$ commutes with any element in $M_{k 0}$ 
by $M_{00}^{\prime} \cap M_{0 l} \subset M_{10}^{\prime} \cap M_{1 l}$, so we get the inclusion $M_{00}^{\prime} \cap M_{0 l}^{\prime} \subset M_{\infty, 0}^{\prime} \cap M_{\infty, l}^{\prime}$.

Take any $x \in M_{\infty, 0}^{\prime} \subset M_{\infty, l}$. Set $x_{k}=E_{M_{l l}}(x) \in M_{k 0}^{\prime} \cap M_{k l}$. The inclusion $M_{00}^{\prime} \cap M_{0 l} \subset M_{2 k, 0}^{\prime} \cap M_{2 k, l}$ implies $M_{00}^{\prime} \cap M_{0 l}=M_{2 k, 0}^{\prime} \cap M_{2 k, l}$ because these two higher relative commutants are isomorphic and finite dimensional. So $x_{2 k} \in M_{2 k, 0}^{\prime} \cap M_{2 k, l}=M_{00}^{\prime} \cap M_{0 l}$ implies $x_{0}=E_{M_{01}}\left(x_{2 k}\right)=x_{2 k}$, and then $x=\lim _{k} x_{2 k}$ $=x_{0} \in M_{00}^{\prime} \cap M_{0 l}$, which is the converse inclusion.

Next $M_{01}^{\prime} \cap M_{0 l} \subset M_{00}^{\prime} \cap M_{0 l}$ implies $M_{01}^{\prime} \cap M_{0 l} \subset M_{01}^{\prime} \cap M_{10}^{\prime} \cap M_{1 l}$, which in turn implies $M_{01}^{\prime} \cap M_{01} \subset M_{11}^{\prime} \cap M_{1 l}$ by [52, Corollary 7.1]. Then the same argument as above shows $M_{\infty, 1}^{\prime} \cap M_{\infty, l}^{\prime}=M_{01}^{\prime} \cap M_{0 l}^{\prime}$.

The subfactors $M_{00} \subset M_{01}$ and $M_{\infty, 0} \subset M_{\infty, 1}$ are conjugate by strong amenability.

Q.E.D.

Lemma 6.3. Suppose that the following square satisfies Assumption 1.1

$$
\begin{array}{ccc}
M_{00} & \subset & M_{01} \\
\cap & & \cap \\
M_{10} & \subset M_{11}
\end{array}
$$

We make a double sequence $\left\{M_{k l}\right\}_{k, l \geq 0}$ of type $I_{1}$ factors and further assume the condition $M_{00}^{\prime} \cap M_{k 0}=M_{01}^{\prime} \cap M_{k 1}$ for $k \geq 0$. Set $S=M_{10}^{\prime} \cap M_{\infty, 0}$ $=\vee_{k \geq 1}\left(M_{10}^{\prime} \cap M_{k 0}\right)$. Then $S^{\prime} \cap M_{\infty, 0}=M_{10}$ and $S^{\prime} \cap M_{\infty, 1}=M_{11}$.

Proof. If $x \in M_{10}^{\prime} \cap M_{k 0}$, then $x \in M_{00}^{\prime} \cap M_{k 0}=M_{01}^{\prime} \cap M_{k 1}$, so $x \in M_{10}^{\prime} \cap M_{01}^{\prime}$ $\cap M_{k 1}=M_{11}^{\prime} \cap M_{k 1}$.

If $x \in M_{11}^{\prime} \cap M_{k 1}$, then $x \in M_{01}^{\prime} \cap M_{k 1}=M_{00}^{\prime} \cap M_{k 0}$, so $x \in M_{10}^{\prime} \cap M_{k 0}$, Thus we have proved $M_{10}^{\prime} \cap M_{k 0}=M_{11}^{\prime} \cap M_{k 1}$, and $S=M_{11}^{\prime} \cap M_{\infty, 1}=\vee_{k \geq 1}\left(M_{11}^{\prime} \cap M_{k 1}\right)$.

Then the conclusion is a consequence of the strong amenability by [44, Theorem 5.3.1 (iv)].

The above two lemmas show the following theorem immediately which gives a relation between the coset construction and a paragroup action on subfactors.

Theorem 6.4. Suppose that the following square satisfies Assumption 1.1

$$
\begin{array}{ccc}
M_{00} & \subset M_{01} \\
\cap & & \cap \\
M_{10} & \subset M_{11}
\end{array}
$$

We make a double sequence $\left\{M_{k l}\right\}_{k, l \geq 0}$ of type $I I_{1}$ factors and further assume the following two conditions.

$$
\begin{aligned}
& M_{00}^{\prime} \cap M_{0 l} \subset M_{10}^{\prime} \cap M_{1 l} \text { for } l \geq 0 . \\
& M_{00}^{\prime} \cap M_{k 0}=M_{01}^{\prime} \cap M_{k 1} \text { for } k \geq 0 .
\end{aligned}
$$

Then there exists a subfactor $S$ of $M_{00}$ such that $S^{\prime} \cap M_{00} \subset S^{\prime} \cap M_{01}$ is 
conjugate to $M_{10} \subset M_{11}$.

We have the following example of Theorem 6.4.

Example 6.5. Take the following square (2) constructed from the triple sequence of the string algebras $\left\{A_{j k}\right\}$. Because the subfactor $M_{00} \subset M_{01}$ is of type $A_{11}$. Condition (I) of Theorem 6.4 is automatically satisfied. Next as in [25, pages 134-135], we can construct $\left\{A_{-1, k, l}\right\}$ for $k \geq 0, l \geq 1$, then the flatness of the Jones projection and the compactness argument [37, II.6] imply $M_{00}^{\prime} \cap M_{k 0}=A_{0 k 0}=A_{-1, k, 1}=M_{01}^{\prime} \cap M_{k 1}$, which is Condition (II) of the Theorem. Thus we know that each of the two subfactors of type $E_{6}$ can be constructed from the subfactor of type $A_{11}$ with the coset construction.

Similarly, each of the two subfactors of type $E_{8}$ can be constructed from the subfactor of type $A_{29}$ with the coset construction. (We can make a similar construction for $A_{17}$, but then the resulting subfactor is of type $D_{10}$, not $E_{7}$. [12])

We have another example of the coset construction.

Example 6.6. Choose a connected, simply connected, compact simple Lie group $G$ with non-trivial center. Let $G$ be a non-trivial subgroup of the center $Z(G)$. Suppose that the level $k$ of the Wess-Zumino-Witten model associated with $G$ satisfies conditions (2), (3) in [63] so that the obstruction for flatness arising from the conformal dimension vanishes. In this case, we have an action $\alpha$ of the finite group $G$ on the subfactor $N \subset M$ obtained form a WZW-model $G_{k}$ as in [6]. Make the following commuting square.

$$
\begin{array}{ccc}
M_{00}=N & \subset & M_{01}=M \\
\cap & & \cap \\
M_{10}=N \times_{\alpha} G & \subset & M_{11}=M \times_{\alpha} G
\end{array}
$$

Because the action $\alpha$ has the trivial Loi invariant by [13, Section 6], we know that Condition (I) of Theorem 6.4 is satisfied. Condition (II) is also satisfied because the both vertical inclusions are crossed products by the same group. Thus the subfactor $N \times_{\alpha} G \subset M \times_{\alpha} G$, which is conjugate to the orbifold subfactor $N^{\alpha} \subset M^{\alpha}$, is obtained from $N \subset M$ with the coset construction.

Thus our orbifold construction in [24], [10], [63] can be regarded also as the coset construction. This also menas that our "crossed products by a paragroup action on a subfactor" can be interpreted as an orbifold construction for a paragroup action. From this viewpoint, Condition (I) of Theorem 6.4 is regarded as representing "approximate innerness" of the paragroup action. 


\section{References}

[1] Alexander, J. W., The combinatorial theory of complexes, Ann, Math., (2) 31 (1930), 294-322.

[2] Andrews, G. E., Baxter, R. J. and Forrester, P. J., Eight vertex SOS model and generalized RogersRamanujan type identities, J. Stat. Phys., 35 (1984), 193-266.

[3] Bisch, D., On the existence of central sequences in subfactors, Trans. Amer. Math. Soc., 321 (1990), 117-128.

[4] , On the structure of finite depth subfactors, "Algebraic methods in operator theory". Birkhäuser (1994), 175-194.

[5] - A note on intermediate subfactors, Pac. J. Math., 163 (1994), 201-216.

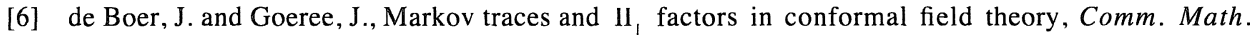
Phys., 139 (1991), 267-304.

[7] Choda, M. and Kosaki, H., Strongly outer actions for inclusion of factors, J. Funct. Anal., 122 (1994), 315-332.

[8] Connes, A., Outer conjugacy classes of automorphisms of factors, Ann. Sci. École Norm. Sup., 8 (1975), 383-419.

[9] Di Francesco, P. and Zuber, J.-B., $S U(N)$ lattice integrable models associated with graphs, Nucl. Phys., B338 (1990), 602-646.

[10] Evans, D. E. and Kawahigashi, Y., Orbifold subfactors from Hecke algebras, Comm. Math. Phys., 165 (1994), 445-484.

[11] - From subfactors to 3-dimensional topological quantum field theories and back, to appear in Internat. J. Math.

[12] - The $E_{7}$ commuting squares produce $D_{10}$ as principal graph, Publ. RIMS, Kyoto Univ., 30 (1994), 151-166.

[13] - Subfactors and conformal field theory, in Quantum and non-commutative analysis, Kluwer Academic (1993), 341-369.

[14] Fendley, P., New exactly solvable orbifold models, J. Phys., A22 (1989), 4633-4642.

[15] Fendley, P. and Ginsparg, P., Non-critical orbifolds, Nucl. Phys., B324 (1989), 549-580.

[16] Goodman, F., de la Harpe, P. and Jones, V. F. R., Coxeter graphs and towers of algebras, MSRI Publications 14, Springer, 1989.

[17] Haagerup, U., Principal graphs of subfactors in the index range $4<[M: N]<3+\sqrt{2}$, Subfactors, World Scientific, (1994) 1-38.

[18] Izumi, M., Application of fusion rules to classification of subfactors, Publ. RIMS, Kyoto Unvi., 27 (1991), 953-994.

[19] - On flatness of the Coxeter graph $E_{8}$, Pac. J. Math., 166 (1994), 305-327.

[20] Jones, V. F. R., Actions of finite groups on the hyperfinite type II, factor, Mem. Amer. Math. Soc., 28 No. 237 (1980), 1-70.

[21] - Index for subfactors, Invent. Math., 72 (1983), 1-15.

[22] - A polynomial invariant for knots via von Neumann algebras, Bull. Amer. Math. Soc., 12 (1985), 103-112.

[23] Kawahigashi, Y., Automorphisms commuting with a conditional expectation onto a subfactor with finite index, J. Operator Theory, 28 (1992), 127-145.

[24] - On flatness of Ocneanu's connections on the Dynkin diagrams and classification of subfactors, J. Funct. Anal., 127 (1995), 63-107.

[25] - Exactly solvable orbifold models and subfactors, in Functional Analysis and Related Topics, Lect. Notes. in Math., Springer Verlag, 1540, (1992), 127-147.

[26] Centrally trivial automorphisms and an analogue of Connes' $\chi(M)$ for subfactors, Duke Math.J., 71 (1993), 93-118.

[27] - Paragroups as quantized Galois groups of subfactors, to appear in Sugaku Exp.

[28] Kirillov A. N. and Reshetikhin, N. Yu., Representations of the algebra $U_{q}\left(s l_{2}\right)$, q-orthogonal polynomials and invariants for links, in Infinite dimensional Lie algebras and groups (V. G. Kac, ed.), Adv. Ser. Math. Phys., 7 (1988), 285-339.

[29] Kosaki, H., Automorphisms in irreducible decompositions of sectors, in Quantum and noncommutative analysis, , Kluwer Academic (1993), 305-316.

[30] Kostov, I., Free field presentation of the $A_{n}$ coset models on the torus, Nucl. Phys., $\mathbb{B} 300$ (1988), $559-587$.

[31] Loi, P. H., On automorphisms of subfactors, preprint 1990.

[32] Moore G., and Seiberg, N., Classical and quantum conformal field theory, Comm. Math. Phys., 123 (1989), 177-254. 
[33] Nitica, V. and Török, A., in preparation.

[34] Ocneanu, A., Actions of discrete amenable groups on factors, Lect. Notes in Math., Springer, Berlin, 1138, (1985).

[35] - Quantized group, string algebras and Galois theory for algebras, in Operator algebras and applications, 2 (Warwick, 1987), London Math. Soc. Lect. Note Series, Cambridge University Press, 136 (1988), 119-172.

[36] - Graph geometry, quantized groups and nonamenable subfactors, Lake Tahoe Lectures, June-July, 1989.

[37] - Quantum symmetry, differential geometry of finite graphs and classification of subfactors, University of Tokyo Seminary Notes 45 , (Notes recorded by Y. Kawahigashi), 1991.

[38] - An invariant coupling between 3-manifolds and subfactors, with connections to topological and conformal quantum field theory, preprint 1991.

[39] Okamoto, S., Invariants for subfactors arising from Coxeter graphs, in Current Topics in Operator Algebras, World Scientific Publishing, (1991), 84-103.

[40] Pimsner, M. and Popa, S., Entropy and index for subfactors, Ann. Scient. Éc. Norm. Sup., 19 (1986), 57-106.

[41] - Iterating the basic constructions, Trans. Amer. Math. Soc., 310 (1988), 127-134.

[42] Popa, S., Correspondences, preprint 1986.

[43] Classification of subfactors: reduction to commuting squares, Invent. Math., 101 (1990), $19-43$.

[44] - Classification of amenable subfactors of type II, Acta Math., 172 (1994), 352-445.

[45] - On the classification of actions of amenable groups on subfactors, C. R. Acad. Sc. Paris., 315 (1992), 295-299.

[46] Classification of actions of discrete amenable groups on amenable subfactors of type II, preprint 1992

[47] - Approximate innerness and central freeness for subfactors: A classification result, Subfactors, World Scientific (1994), 274-293.

[48] Classification of subfactors of finite depth of the hyperfinite type III factor, to appear in C. R. Acad. Sc. Paris.

[49] Reshetikhin, N. Yu. and Turaev, V. G., Invariants of 3-manifolds via link polynomials and quantum groups, Invent. Math., 103 (1991), 547-597.

[50] Roche, Ph., Ocneanu cell calculus and integrable lattice models, Comm. Math. Phys., 127 (1990), 395-424.

[51] Sano, T., Commuting co-commuting squares and finite dimensional Kac algebras, preprint 1993.

[52] Sano, T. and Watatani, Y., Angles between two subfactors, J. Operator Theory, 32 (1994), 209-241.

[53] Schou, J., Commuting squares and index for subfactors, Ph. D. Thesis. Odense University, 1990.

[54] Sunder, V.S. and Vijayarajan, A. K., On the non-occurrence of the Coxeter graphs $\beta_{2 n+1}, E_{7}, D_{2 n+1}$ as principal graphs of an inclusion of II, factors, Pac. J. Math., 161 (1993), 185-200.

[55] Turaev, V. G. and Viro, O. Y., State sum invariants of 3-manifolds and quantum 6j-symbols, Topology, 31 (1992), 865-902.

[56] Wierzbicki, J., An estimate of the depth from an intermediate subfactor, preprint 1993.

[57] Wierzbicki, J. and Watatani, Y., Commuting squares and relative entropy for two subfactors, preprint 1992.

[58] Wenzl, H., Hecke algebras of type $A_{n}$ and subfactors, Invent. Math., 92 (1988), 345-383.

[59] Winsløw, C., Approximately inner "automorphisms on inclusions of type III $_{\lambda}$ factors, Pac. J. Math., 166 (1994), 385-400.

[60] - Strongly free actions on subfactors, Internat. J. Math., 4 (1993), 675-688.

[61] - Crossed products of $\mathrm{II}_{1}$-subfactors by strongly outer actions, Proc. Amer. Math. Soc., 347 (1995), 985-991.

[62] Witten, E., Quantum field theory and Jones polynomial, Comm. Math. Phys., 121 (1989), 351-399.

[63] Xu, F., Orbifold construction in subfactors, Comm. Math. Phys., 116 (1994), 237-254.

[64] - The flat parts of non-flat orbifolds, to appear in Pac. J. Math.

[65] Yamagami, Y., A note on Ocneanu's approach to Jones' index theory, Internat. J. Math., 4 (1993), 859-871. 
